\title{
Sensitivity of the IceCube Detector to Astrophysical Sources of High Energy Muon Neutrinos
}

J. Ahrens ${ }^{\circ}$, J.N. Bahcall ${ }^{\text {h }}$, X. Bai ${ }^{\text {a }}$, R.C. Bay ${ }^{\text {n }}$, T. Becka ${ }^{\circ}$, K.-H. Becker ${ }^{b}$, D. Berley ${ }^{\mathrm{p}}$, E. Bernardini ${ }^{\mathrm{f}}$, D. Bertrand ${ }^{\mathrm{c}}$,

D.Z. Besson ${ }^{i}$, E. Blaufuss ${ }^{\text {p }}$, D.J. Boersma ${ }^{f}$, S. Böser ${ }^{f}$, C. Bohm ${ }^{\mathrm{z}}$, O. Botner ${ }^{\mathrm{x}}, \mathrm{A}$. Bouchta ${ }^{\mathrm{x}}$, O. Bouhali ${ }^{\mathrm{c}}$, T. Burgess ${ }^{z}$, W. Carithers ${ }^{j}$, T. Castermans ${ }^{r}$, J. Cavin ${ }^{\mathrm{v}}$, W. Chinowsky ${ }^{\mathrm{j}}$, D. Chirkin ${ }^{\mathrm{n}}$, B. Collin ${ }^{\ell}$, J. Conrad ${ }^{\mathrm{x}}$, J. Cooley ${ }^{\mathrm{u}}$, D.F. Cowen ${ }^{\ell, \mathrm{k}}$, A. Davour ${ }^{\mathrm{x}}$, C. De Clercq ${ }^{\mathrm{a}}$, T. DeYoung ${ }^{\mathrm{p}}$, P. Desiati ${ }^{\mathrm{u}}$, R. Ehrlich ${ }^{\mathrm{p}}$, R.W. Ellsworth ${ }^{\mathrm{q}}$, P.A. Evenson ${ }^{a}$, A.R. Fazely ${ }^{\mathrm{m}}$, T. Feser ${ }^{\mathrm{o}}$, T.K. Gaisser ${ }^{\mathrm{a}}$, J. Gallagher ${ }^{\mathrm{t}}$, R. Ganugapati ${ }^{\mathrm{u}}, \mathrm{H}$. Geenen ${ }^{\mathrm{b}}$, A. Goldschmidt ${ }^{\mathrm{j}}$, J.A. Goodman $^{\mathrm{p}}$, R.M. Gunasingha ${ }^{\mathrm{m}}$, A. Hallgren ${ }^{\mathrm{x}}$, F. Halzen ${ }^{\mathrm{u}}$,

K. Hanson ${ }^{\text {u }}$, R. Hardtke ${ }^{\text {, }}$ T. Hauschildt ${ }^{\mathrm{f}}$, D. Hays ${ }^{\mathrm{j}}$, K. Helbing ${ }^{j}$, M. Hellwig ${ }^{\circ}$, P. Herquet ${ }^{r}$, G.C. Hill ${ }^{\mathrm{u}}$, D. Hubert ${ }^{\text {aa }}$, B. Hughey ${ }^{\text {u }}$, P.O. Hulth ${ }^{z}$, K. Hultqvist ${ }^{\text {, }}$, S. Hundertmark ${ }^{z}$, J. Jacobsen ${ }^{j}$, G.S. Japaridze ${ }^{d}$, A. Jones ${ }^{j}$, A. Karle ${ }^{\text {u }}$, H. Kawai ${ }^{\mathrm{e}}$, M. Kestel $^{\ell}$, N. Kitamura ${ }^{\mathrm{v}}$, R. Koch ${ }^{ }$, L. Köpke ${ }^{\circ}$, M. Kowalski ${ }^{f}$, J.I. Lamoureux ${ }^{j}$, H. Leich ${ }^{f}$, I. Liubarsky ${ }^{\mathrm{g}}$, J. Madsen ${ }^{\mathrm{w}}$, H.S. Matis ${ }^{\mathrm{j}}$, C.P. McParland ${ }^{\mathrm{j}}$, T. Messarius ${ }^{b}$, P. Mészáros ${ }^{\mathrm{k}, \ell}$, Y. Minaeva ${ }^{\mathrm{z}}$, R.H. Minor ${ }^{\mathrm{j}}$, P. Miočinovićn ${ }^{n}, H$. Miyamoto ${ }^{\mathrm{e}}$, R. Morse $^{\mathrm{u}}$, R. Nahnhauer ${ }^{\mathrm{f}}$, T. Neunhöffer ${ }^{\circ}$, P. Niessen ${ }^{\text {aa }}$, D.R. Nygren ${ }^{j}$, H. Ögelman ${ }^{u}$, Ph. Olbrechts ${ }^{\text {aa }}$, S. Patton ${ }^{j}$, R. Paulos ${ }^{\text {u, }}$

C. Pérez de los Heros ${ }^{\mathrm{x}}$, A.C. Pohl ${ }^{\mathrm{z}}$, J. Pretz ${ }^{\mathrm{p}}$, P.B. Price ${ }^{\mathrm{n}}$, G.T. Przybylski ${ }^{j}$, K. Rawlins ${ }^{\mathrm{u}}$, S. Razzaque ${ }^{\mathrm{k}}$, E. Resconi ${ }^{\mathrm{f}}$,

W. Rhode ${ }^{\mathrm{b}}$, M. Ribordy ${ }^{\mathrm{r}}$, S. Richter ${ }^{\mathrm{u}}$, H.-G. Sander ${ }^{ }$, K. Schinarakis ${ }^{b}$, S. Schlenstedt ${ }^{f}$, D. Schneider ${ }^{u}$, R. Schwarz ${ }^{u}$,

D. Seckel ${ }^{\text {a }}$, A.J. Smith ${ }^{\mathrm{p}}$, M. Solarz ${ }^{\mathrm{n}}$, G.M. Spiczak ${ }^{\mathrm{w}}$, C. Spiering ${ }^{f}$, M. Stamatikos ${ }^{u}$, T. Stanev ${ }^{\text {a }}$, D. Steele ${ }^{u}$,

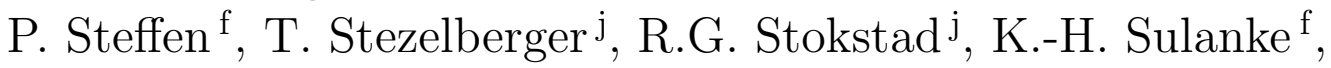


G.W. Sullivan ${ }^{\text {p, T.J. Sumner }}{ }^{\mathrm{g}}$, I. Taboada ${ }^{\mathrm{s}}$, S. Tilav ${ }^{\mathrm{a}}$, N. van Eijndhoven ${ }^{\mathrm{y}}$, W. Wagner ${ }^{\mathrm{b}}$, C. Walck ${ }^{\mathrm{z}}$, R.-R. Wang ${ }^{\mathrm{u}}$, C.H. Wiebusch ${ }^{\mathrm{b}}$, C. Wiedemann ${ }^{\mathrm{z}}$, R. Wischnewski ${ }^{\mathrm{f}}$, H. Wissing ${ }^{\mathrm{f}}$, K. Woschnagg ${ }^{\mathrm{n}}$, S. Yoshida ${ }^{\mathrm{e}}$

${ }^{a}$ Bartol Research Institute, University of Delaware, Newark, DE 19716, USA

${ }^{\mathrm{b}}$ Fachbereich 8 Physik, BUGH Wuppertal, D-42097 Wuppertal, Germany

${ }^{\mathrm{c}}$ Université Libre de Bruxelles, Science Faculty CP230, Boulevard du Triomphe, B-1050 Brussels, Belgium

${ }^{\mathrm{d}}$ CTSPS, Clark-Atlanta University, Atlanta, GA 30314, USA

${ }^{\mathrm{e}}$ Dept. of Physics, Chiba University, Chiba 263-8522 Japan

${ }^{\mathrm{f}}$ DESY-Zeuthen, D-15738 Zeuthen, Germany

${ }^{g}$ Blackett Laboratory, Imperial College, London SW7 2BW, UK

${ }^{\mathrm{h}}$ Institute for Advanced Study, Princeton, NJ 08540, USA

${ }^{\mathrm{i}}$ Dept. of Physics and Astronomy, University of Kansas, Lawrence, KS 66045, $U S A$

${ }^{j}$ Lawrence Berkeley National Laboratory, Berkeley, CA 94720, USA

${ }^{\mathrm{k}}$ Dept. of Astronomy and Astrophysics, Pennsylvania State University, University Park, PA 16802, USA

${ }^{\ell}$ Dept. of Physics, Pennsylvania State University, University Park, PA 16802, $U S A$

${ }^{\mathrm{m}}$ Dept. of Physics, Southern University, Baton Rouge, LA 70813, USA

${ }^{\mathrm{n}}$ Dept. of Physics, University of California, Berkeley, CA 94720, USA

${ }^{\circ}$ Institute of Physics, University of Mainz, Staudinger Weg 7, D-55099 Mainz, Germany

${ }^{\mathrm{p}}$ Dept. of Physics, University of Maryland, College Park, MD 20742, USA

${ }^{\mathrm{q}}$ Dept. of Physics, George Mason University, Fairfax, VA 22030 USA

${ }^{\mathrm{r}}$ University of Mons-Hainaut, 7000 Mons, Belgium

${ }^{\mathrm{s}}$ Departamento de Física, Universidad Simón Bolívar, Caracas, 1080, Venezuela

${ }^{\mathrm{t}}$ Dept. of Astronomy, University of Wisconsin, Madison, WI 53706, USA

u Dept. of Physics, University of Wisconsin, Madison, WI 53706, USA

${ }^{\vee}$ SSEC, University of Wisconsin, Madison, WI 53706, USA

${ }^{\mathrm{w}}$ Physics Dept., University of Wisconsin, River Falls, WI 54022, USA

${ }^{x}$ Division of High Energy Physics, Uppsala University, S-75121 Uppsala, Sweden

${ }^{\mathrm{y}}$ Faculty of Physics and Astronomy, Utrecht University, NL-3584 CC Utrecht, The Netherlands

${ }^{\mathrm{z}}$ Dept. of Physics, Stockholm University, SE-10691 Stockholm, Sweden

${ }^{\text {aa } V r i j e ~ U n i v e r s i t e i t ~ B r u s s e l, ~ D i e n s t ~ E L E M, ~ B-1050 ~ B r u s s e l s, ~ B e l g i u m ~}$ 


\begin{abstract}
We present the results of a Monte-Carlo study of the sensitivity of the planned IceCube detector to predicted fluxes of muon neutrinos at $\mathrm{TeV}$ to $\mathrm{PeV}$ energies. A complete simulation of the detector and data analysis is used to study the detector's capability to search for muon neutrinos from sources such as active galaxies and gamma-ray bursts. We study the effective area and the angular resolution of the detector as a function of muon energy and angle of incidence. We present detailed calculations of the sensitivity of the detector to both diffuse and pointlike neutrino emissions, including an assessment of the sensitivity to neutrinos detected in coincidence with gamma-ray burst observations. After three years of datataking, IceCube will have been able to detect a point source flux of $E_{\nu}^{2} \times d N_{\nu} / d E_{\nu}=$ $7 \times 10^{-9} \mathrm{~cm}^{-2} \mathrm{~s}^{-1} \mathrm{GeV}$ at a 5-sigma significance, or, in the absence of a signal, place a $90 \%$ c.l. limit at a level $E_{\nu}^{2} \times d N_{\nu} / d E_{\nu}=2 \times 10^{-9} \mathrm{~cm}^{-2} \mathrm{~s}^{-1} \mathrm{GeV}$. A diffuse $E^{-2}$ flux would be detectable at a minimum strength of $E_{\nu}^{2} \times d N_{\nu} / d E_{\nu}=$ $1 \times 10^{-8} \mathrm{~cm}^{-2} \mathrm{~s}^{-1} \mathrm{sr}^{-1} \mathrm{GeV}$. A gamma-ray burst model following the formulation of Waxman and Bahcall would result in a 5-sigma effect after the observation of 200 bursts in coincidence with satellite observations of the gamma-rays.
\end{abstract}

Key words: Neutrino detectors

PACS: 95.55.Vj, 95.85.Ry

\title{
1 Introduction
}

The emerging field of high-energy neutrino astronomy $[1,2,3]$ has seen the construction, operation and results from the first detectors, and proposals for the next generation of such instruments. The first pioneering efforts of the DUMAND [4] collaboration were followed by the successful deployments of NT-200 at Lake Baikal [5] and AMANDA [6] at the South Pole. These detectors have demonstrated the feasibility of large neutrino telescopes in open media like water or ice. They have observed neutrinos produced in the atmosphere [7] and have put limits on the flux of extraterrestrial neutrinos [8,9], which are significantly below those obtained from the much smaller underground neutrino detectors $[10,46]$. The results obtained so far, and further refinements to astrophysical theories of extra-terrestrial neutrino fluxes from cosmic sources have provided the impetus to construct a neutrino observatory on a much larger scale. Proposals for a detector in the deep water of the Mediterranean have come from the ANTARES [12], NESTOR [13] and NEMO [14] collaborations. IceCube is a projected cubic kilometer under ice neutrino detector $[15,16,17]$, to be located near the geographic South Pole in Antarctica. 
The IceCube detector will consist of optical sensors deployed at depth into the thick Polar ice sheet. The ice will serve as Cherenkov medium for secondary particles produced in neutrino interactions in or around the instrumented volume. The successful deployment and operation of the AMANDA detector have shown that the Polar ice sheet is a suitable medium for a large neutrino telescope and the analysis of the AMANDA data proves the science potential of such a detector.

IceCube will offer great advantages over AMANDA beyond its larger size: It will have a higher efficiency and a better angular resolution in reconstructing tracks, it will map electromagnetic and hadronic showers (cascades) from electron- and tau-neutrino interactions and, most important, it will have a superior energy resolution. Simulations, backed by AMANDA data, indicate that the direction of muons can be determined with sub-degree accuracy and their energy measured to better than $30 \%$ in the logarithm of the energy. The direction of electron neutrinos that have produced electromagnetic cascades will be reconstructed to better than $25^{\circ}$ and the response in energy is linear with a resolution better than $10 \%$ in the logarithm of the energy [17]. Energy resolution is critical because no atmospheric muon or neutrino background exceeds $1 \mathrm{PeV}$ in a cubic-kilometer detector and full sky coverage is achieved.

IceCube will be able to investigate a large variety of scientific questions in astronomy, astrophysics, cosmology and particle physics $[17,23]$. In this paper we will focus on the IceCube performance in searching for $\mathrm{TeV}$ to $\mathrm{PeV}$ muon neutrinos, as expected from sources such as active galactic nuclei, gamma-ray bursts or other cosmic accelerators observed as $\mathrm{TeV}$ gamma ray emitters. We will present the results of a Monte Carlo study, including the simulation of the detector and the full analysis chain from filtering of the triggered data to event reconstruction and selection. We will assess basic detector parameters like the pointing resolution and the effective area of the detector directly from simulated data. Further, we present a detailed calculation of the sensitivity of the detector to both diffuse and point-like neutrino emission following generic energy spectra, providing a benchmark sensitivity for some of the fundamental goals of high energy neutrino astronomy.

\section{The IceCube Detector}

The IceCube detector is planned as a cubic kilometer successor to the AMANDA detector, currently operated at the South Pole. It will consist of 4800 photomultiplier tubes (PMTs) of 10 inch diameter, each enclosed in a transparent pressure sphere. These optical modules (OMs) will be deployed into vertical holes drilled to a depth of $2400 \mathrm{~m}$ with pressurized hot water. As a significant improvement over the AMANDA technology, each OM will also house the 


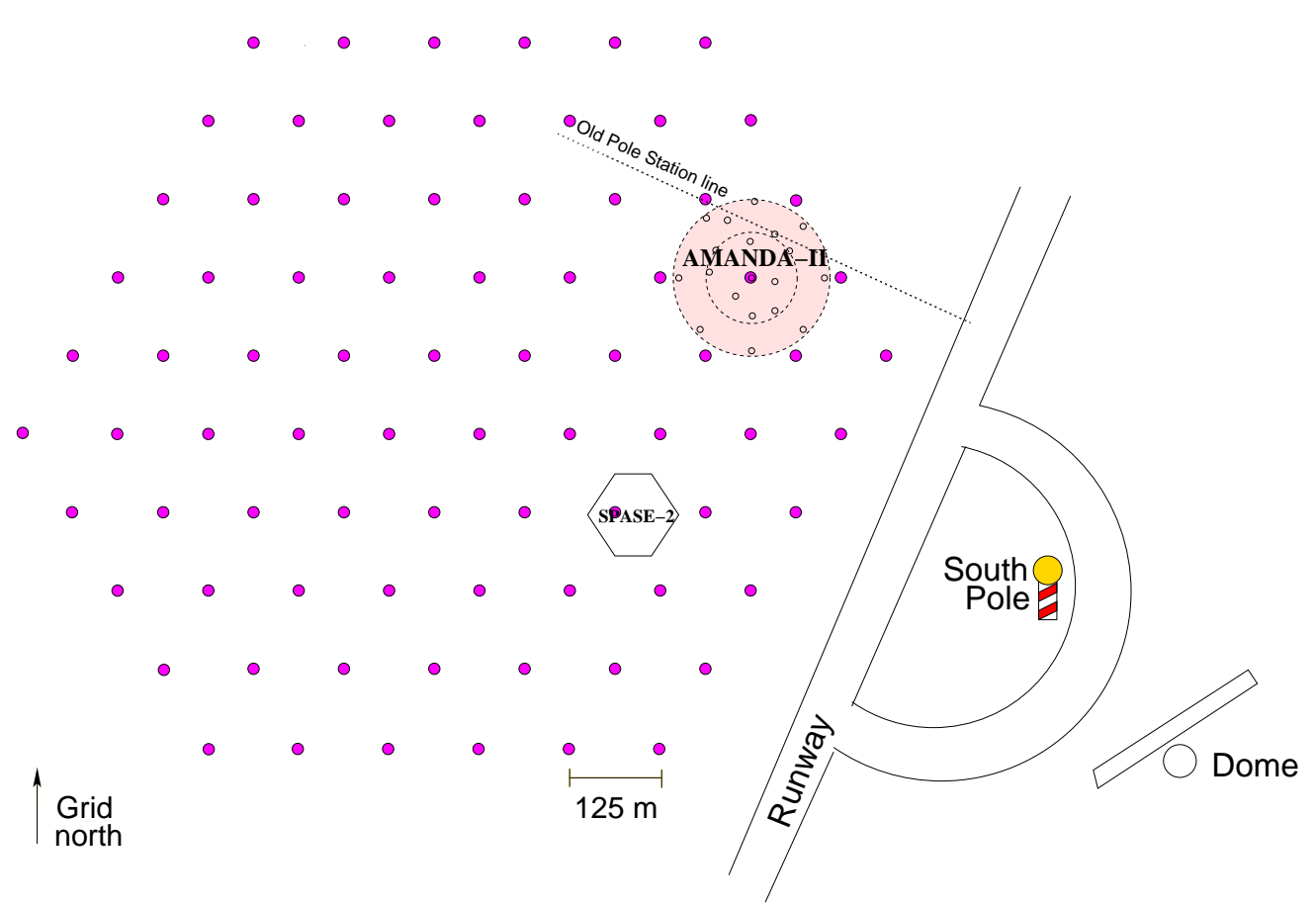

Fig. 1. Schematic diagram of the arrangement of the strings of the IceCube detector at the South Pole station. The existing AMANDA detectors and the SPASE air shower array will be embedded in the new detector.

electronics to digitize the PMT pulses, retaining the full waveform information $[17,18]$. The digitized signals will be transmitted by twisted pair cables to the surface data acquisition system. Adjacent OMs will be connected via a separate twisted pair cable, which makes possible a local level-one hardware trigger in the ice. Local triggers will be combined by surface processors to form a global trigger. Triggered events will be filtered and reconstructed on-line, and the relevant information will be transmitted via satellite to the northern hemisphere.

The dark noise rate of an OM will be $300-500 \mathrm{~Hz}$. This low rate is due to the sterile and low temperature environment of the deep Antarctic ice. The absorption length of light from UV to blue varies between $50 \mathrm{~m}$ and $150 \mathrm{~m}$, depending on depth. This allows photons to be collected even from very distant tracks. In fact, many of the photons will be delayed by scattering in the ice, but the some trajectory information may be retrieved from the dispersedsignal PMT pulse shapes. The single-pulse timing accuracy will be about $5 \mathrm{~ns}$, the sampling period 2-3 ns and the double pulse resolution close to $6 \mathrm{~ns}$. The dynamic range of one PMT will be about 200 photo-electrons per $15 \mathrm{~ns}$, and several thousand photo-electrons integrated over the full waveform from a distant light source.

A central feature of the system is automatic time and amplitude calibration, critical for a large array at remote location. Each OM will be equipped with 
a set of bright $370 \mathrm{~nm}$ LED pulsers. The LED signals, an essential part of the calibration system, can be seen by OMs as far away as $200 \mathrm{~m}$ from their source LEDs.

The OMs will be deployed on 80 vertical strings, each carrying 60 OMs spaced by $17 \mathrm{~m}$. The instrumented volume will span a depth ranging from $1400 \mathrm{~m}$ to $2400 \mathrm{~m}$ below the ice surface. The strings will be arranged in a triangular pattern such that the distances between each string and its six nearest neighbors are $125 \mathrm{~m}$. This configuration is the result of an extensive optimization procedure $[19,20]$, covering arrays with the number of OMs ranging from 2400 (half the design number) to 9600 (twice the design number), with equally spaced strings, nested sub-arrays of larger density, and a variety of "exotic" configurations. A schematic sketch of the spatial string arrangement is shown in figure 1. The complete detector will be operational perhaps as soon as five years after the start of construction, but during construction all deployed strings will produce high quality data for the collaboration to analyze.

The IceCube array deep in the ice will be complemented by IceTop, a surface air shower array consisting of a set of 160 frozen water tanks. The tanks will be arranged in pairs, separated by a few meters, on top of each IceCube string. IceTop is the logical extension of the SPASE surface array [21] which already is a unique asset for AMANDA. The data of the air-shower parameters measured at the surface combined with the signal of the high energy muon component under-ice provide a new measure for the primary composition of cosmic rays. Furthermore, IceTop will serve as a veto for air-shower-induced background and it will provide cross checks for the detector geometry calibration, absolute pointing accuracy and angular resolution. In addition, the energy deposited by tagged muon bundles in air-shower cores will be an external source for energy calibration.

\section{Simulation and Analysis Chain}

The science potential of a kilometer scale neutrino telescope has been assessed in previous papers by convoluting the expected neutrino induced muon flux from various astrophysical sources with an assumed square kilometer effective detector area $[22,23,24]$. In this work we use a full simulation of the event triggering, reconstruction and data selection to assess the detector capabilities. The simulation of the detector and the analysis of the Monte Carlo data rely on the software packages presently provided by the AMANDA collaboration $[25,40]$. This means that the software concepts and analysis techniques used here have proven capable and have been verified by real data taken with the AMANDA detector. However, a full simulation of the IceCube hardware was not possible with the present software: The simulated data correspond to the 
original AMANDA read-out, which does not yield the PMT waveforms. Advanced analysis methods which take advantage of this additional information were not applied and hence we may yield a conservative picture of the IceCube performance.

A brief outline of the different stages in the used processing chain is given in the following sections.

\subsection{Event Generation}

The backgrounds for searches for extraterrestrial neutrinos come from the decay of mesons produced from cosmic ray (CR) interactions in the atmosphere. The decay products include both muons and neutrinos. The muons will be responsible for the vast majority of triggers, since they are very penetrating and are thus capable to reach the detector depth. Air-shower induced events can be identified by the fact that they involve exclusively down-going tracks and a comparatively small deposit of Cherenkov light in the detector, as the muons will have lost most of their energy upon reaching the detector. However, an up-going track might be faked if two uncorrelated air-showers produce time-coincident muons within the detector. In fact, about three percent of all triggered events will be caused by muons from two independent air-showers.

The simulation packages Basiev [26] and Corsika [27] were used for the CR induced muon background. Roughly 2.4 million events containing muon tracks from one single air shower (Atm $\mu^{\text {single }}$ ) were simulated with primary energies up to $10^{8} \mathrm{GeV}$. High energy events as well as events that contain tracks close to the horizon were oversampled, in order to achieve better statistics at high analysis levels. In addition, we have simulated one million events containing tracks from two independent air-showers (Atm $\left.\mu^{\text {double }}\right)$.

The muons induced by atmospheric neutrinos (Atm $\nu$ ) form a background over $4 \pi \mathrm{sr}$ and up to very high energies. However, the energy spectrum of atmospheric neutrinos falls steeply like $d N_{\nu} / d E_{\nu} \propto E_{\nu}^{-3.7}$, whereas one expects an energy spectrum as hard as $E^{-2}$ from shock acceleration mechanisms in anticipated cosmic TeV-neutrino sources. Therefore, cosmic neutrino energies should extend to higher values and cause more light in the detector than will atmospheric neutrinos. The amount of light observed in an event is therefore useful as a criterion to separate high energy muons induced by cosmic neutrinos from those induced by atmospheric neutrinos. An uncertainty in the flux of atmospheric neutrinos arises from the poorly known contribution of prompt decays of charmed mesons produced in the atmosphere [28,29].

Neutrino induced events have been simulated with the program nusim [30]. Neutrinos are sampled from a $1 / E$ spectrum and are later re-weighted to pro- 
duce different energy spectra as required. The code includes a simulation of the neutrino propagation through the Earth, taking into account absorption in charged current interactions as well as neutral current regeneration. The neutrino cross sections are calculated using the MRSG [31] parton distributions. The column density of nucleons to be traversed is calculated according to the Preliminary Reference Earth Model [32]. Muons that are produced in the rock beneath the detector are propagated to the rock/ice boundary using the Lipari-Stanev [33] muon propagation code. In total we have simulated $7.4 \cdot 10^{5}$ events induced by neutrinos with primary energies up to $10^{8} \mathrm{GeV}$. The flatness of the simulated $1 / E$ neutrino spectrum leads to an oversampling of events at high energies for most of the energy spectra investigated.

For "conventional" flux of atmospheric neutrinos (i.e. the component related to decays of pions and kaons) we apply the prediction calculated by Lipari [34]. For the prompt charm contribution we compare two different estimates: The "Recombination Quark Parton Model" (rqpm) developed by Bugaev et al. [35] and the model by Thunman et al. (TIG) [36]. The latter model predicts a substantially smaller rate and may serve as a lower limit for the prompt charm contribution.

For the flux of extraterrestrial neutrinos (Cosmic $\nu$ ) we apply a generic $E^{-2}$ energy spectrum, as expected from shock acceleration. We use a source strength of $E_{\nu}^{2} \times d N_{\nu} / d E_{\nu}=10^{-7} \mathrm{~cm}^{-2} \mathrm{~s}^{-1} \mathrm{sr}^{-1} \mathrm{GeV}$ as a benchmark diffuse flux of extraterrestrial neutrinos. This is an order of magnitude below present experimental limits set on the flux of muon neutrinos [9] or electron neutrinos [37].

\subsection{Muon Propagation}

The propagation of muons through the ice is modeled with either the code by

Lohmann, Kopp and Voss [38] (for muon energies smaller than $10^{5.5} \mathrm{GeV}$ ) or the code by Lipari and Stanev [33] (for muon energies greater than $10^{5.5} \mathrm{GeV}$ ). These codes calculate the stochastic radiative and nuclear interactive energy losses along the muon track within or close to the detector instrumented volume.

The complete tracking of all Cherenkov photons produced by the muon and associated stochastic radiative energy losses for each event would require an impractical amount of computing power. Therefore, the photon amplitudes and timing distributions at all points in space from both a muon and an electromagnetic cascade are pre-calculated and tabulated into fast lookup tables using the PTD [39] software package. This simulation takes into account the scattering and absorption properties of the ice as well as the response of the 
PMT.

\subsection{Detector Simulation}

The response of the entire array of PMTs is modeled with the detector simulation amasim [40,41]. The actual number of photons at an OM is found by sampling from a Poisson distribution with a mean amplitude computed by summing over all contributing muons and cascades. The arrival times of these photons are sampled from the pre-tabulated distributions. Noise photons are added assuming a PMT noise rate of $500 \mathrm{~Hz}$. For the trigger we use a local coincidence filter, requiring 5 local coincidences within a time window of $7 \mu \mathrm{s}$, where a local coincidence is defined as the registration of at least two signals in two or more PMTs within a group of five neighboring OMs on a string.

The detector geometry used in this simulation differs from the finalized design in the total number of strings (we have simulated a 75 string detector instead of 80), the total number of OMs (4575 instead of 4800), the string length (960 $\mathrm{m}$ instead of $1000 \mathrm{~m}$ ) and the location of the detector center (which was simulated at $2000 \mathrm{~m}$, while it will lie at $1800 \mathrm{~m}$ depth in the updated design). The spatial arrangement of the strings in a $125 \mathrm{~m}$ spaced triangular grid is in accordance to what is presented in the previous section.

\subsection{Event Reconstruction}

The triggered events are first filtered based on three fast "first guess" algorithms which use the arrival times of the photons or the topology of OMs having registered a photon signal (or "hit"): (1) The line fit $(L F)$ is based on a simple analytic $\chi^{2}$ minimization [42]. It fits the free parameters (vertex position and velocity) of a hypothetical straight line trajectory to the one-dimensional projection of an observed pattern of hits. (2) The dipole approximation [43] is based on the hit topology: The sum of all unit vectors pointing from one hit to the next in time gives a "dipole vector" $\vec{M}$. The direction of $\vec{M}$ is correlated to the direction of the incoming track(s), while its absolute value is a measure of the goodness of the approximation. (3) The direct walk algorithm $(D W)[43]$ posits as track hypotheses the straight line connections between every two hits that have occurred in separate OMs with a time difference consistent with muon flight time between these two OMs. Those track hypotheses that pass a consistency check with respect to the complete hit pattern of the event are combined to obtain an estimate of the track parameters.

Following this first guess methods, the events are reconstructed using a full 
maximum likelihood reconstruction $(L R)[43,44]$. The probabilities in the likelihood function follow the arrival time distribution of photons emitted along a track as a function of distance and angle of the track with respect to the OM. These distributions have been obtained from a photon propagation simulation. The reconstruction used here relies on the information carried by the first photon that arrives at the PMT. This corresponds to the original AMANDA read-out that only yields the timing information of the PMT pulses. ${ }^{1}$

\section{Basic Performance Capabilities}

The detector trigger rate for a five-fold local coincidence trigger was found to be $1.7 \mathrm{kHz}$. This includes a $50 \mathrm{~Hz}$ rate of triggers due to non-correlated time-coincident air-showers (Atm $\mu^{\text {double }}$ ). As described below, a basic set of standard event selection criteria was established that remove the bulk of the down-going CR induced muons, but still yield a large passing rate for muons from atmospheric neutrinos. These atmospheric neutrinos would then form the background to searches for cosmic neutrinos. We use this level of data reduction as a baseline performance measure.

\subsection{Event Selection}

The most vital criteria to reject the background of downgoing CR induced muons are the zenith angles obtained from various reconstruction and filter algorithms $\left(\Theta_{L R}, \Theta_{L F}, \Theta_{D W}\right.$ and $\left.\Theta_{\vec{M}}\right)$. The easiest way to reject CR muons would be to select exclusively up-going tracks. However, muons from $\mathrm{PeV}$ or EeV neutrino interactions are expected to arrive from directions close to or above the horizon, so it is worthwhile to combine the angular cut with an energy criterion. If the neutrino interaction does not occur far from the detector, the energy deposit of the daughter muon will be high enough to distinguish it from low energy CR muons. An estimator of this energy deposit is the number of OMs (or "channels") that have registered a hit. We therefore accept downgoing tracks provided the channel multiplicity $\left(N_{\mathrm{ch}}\right)$ of the event is sufficiently large.

The individual selection criteria are listed in table 1 . The first three criteria are based on the track directions obtained from the three "first guess" methods

1 In contrast, the IceCube electronics will retain the full pulse shape. Detailed hit information can be extracted from the integrated charge and the peak structure of the pulse. Future reconstructions will therefore profit from the additional information formed by consecutively arriving photons which were multiple-scattered and delayed on their way from the muon track to the PMT. 
and aim at the early rejection of low energy downgoing CR muons. The level of data reduction achieved with the application of cuts 1-3 will be referred to as "level 1".

"Level 2", defined by cuts 4-9, is based on the observables of the more accurate (and more CPU intensive) likelihood reconstruction:

- Events that are reconstructed with zenith angles smaller than $85^{\circ}$ (i.e. directions more than five degrees above the horizon) are rejected, as long as $N_{\text {ch }}$ is smaller than 150. The $N_{\text {ch }}$-criterion is tightened with decreasing zenith angle $\left(\Theta_{L R}\right)$ [cut 4$]$.

Apart from the direction criterion, the likelihood reconstruction provides a series of quality parameters, which we apply in order to select a sample of high quality and well reconstructed events:

- We require the reduced likelihood $(\mathcal{L})$ of the likelihood reconstruction to be sufficiently small. $\mathcal{L}$ is given by the negative logarithm of the likelihood of the best-fit track hypothesis divided by the number of degrees of freedom of the fit, hence a small value indicates a good track quality [cut 5].

- We require a minimum number of direct hits $\left(N_{\text {direct }}\right)$, i.e. hits that have occurred with a sufficiently small delay $(<150 \mathrm{~ns})$ relative to the arrival time predicted for an unscattered Cherenkov photon emitted from the reconstructed track [cut 6].

- We require a minimum track length $(L)$, i.e. a minimum distance along the reconstructed track over which the hits were detected. We define this length as the maximum distance of two hit positions projected on the straight line defining the track direction. A more stringent criterion is a lower bound on the track length using direct hits $\left(L_{\text {direct }}\right)$ [cut 7$]$.

- The consistency of the fitted track direction is checked with the smoothness parameter $[7,43]$. It is a measure of the evenness of the projection of the hit positions along the track, based on a Kolmogorov-Smirnov test. The smoothness parameter can be calculated with either all hits $(S)$, or exclusively direct hits $\left(S_{\text {direct }}\right)$ [cut 8$]$.

- For high quality tracks, the various reconstruction methods are likely to produce similar results close to the original (i.e. true) track. We therefore require the difference in zenith angles obtained by two different methods to be small [cuts 9 and 3].

These quality criteria are particularly important for muons that travel a short path through the instrumented detection volume, e.g. low energy muons or muons that pass only through the outer rim of the detector or even outside its geometrical volume. These muons will cause signals in fewer OMs and therefore leave less information for the reconstruction. Most of the quality 
Table 1

Definition of cuts levels.

\begin{tabular}{|c|c|c|c|}
\hline & Parameter & Cut & Explanation \\
\hline \multicolumn{4}{|c|}{ Level 1: } \\
\hline 1. & $\Theta_{L F}$ & $>60^{\circ}$ if $N_{\mathrm{ch}}<50$ & $\begin{array}{l}\text { zenith angle criterion } \\
\text { based on } L F \text {, applied for } \\
\text { low multiplicity events }\end{array}$ \\
\hline 2. & $\Theta_{\vec{M}}$ & $>50^{\circ}$ if $|\vec{M}|>0.2$ & $\begin{array}{l}\text { zenith angle criterion } \\
\text { based on } \vec{M} \text {, applied for } \\
\text { high goodness-of-fit values }\end{array}$ \\
\hline & $\left|\Theta_{D W}-\Theta_{\vec{M}}\right|$ & $<50^{\circ}$ & consistency of $L F$ and $D W$ \\
\hline \multicolumn{4}{|c|}{ Level 2: } \\
\hline 4. & $\Theta_{L R}$ & $\begin{array}{c}>85^{\circ} \\
\text { or } \\
N_{\mathrm{ch}}>150+250 * \cos \left(\Theta_{L R}\right)\end{array}$ & $\begin{array}{l}\text { zenith angle criterion of } L R \\
\text { which is weakened with } \\
\text { increasing channel multiplicity }\end{array}$ \\
\hline 5. & $\mathcal{L}$ & $<10$ & reduced likelihood of $L R$ \\
\hline 6. & $N_{\text {direct }}$ & $>10$ if $N_{\mathrm{ch}}<50$ & $\begin{array}{l}\text { requirement of } 10 \text { direct hits } \\
\text { for low multiplicity events }\end{array}$ \\
\hline 7. & $\begin{array}{c}L \\
L_{\text {direct }} \\
\end{array}$ & $\begin{array}{c}>300 \mathrm{~m} \\
\text { and } \\
>300 \mathrm{~m} \text { if } N_{\mathrm{ch}}<150 \\
\end{array}$ & $\begin{array}{l}\text { requirement of minimum track } \\
\text { length, using direct hits for } \\
\text { multiplicities smaller than } 150\end{array}$ \\
\hline 8. & $\begin{array}{c}|S| \\
\left|S_{\text {direct }}\right| \\
\end{array}$ & $\begin{array}{c}<0.5 \\
\text { and } \\
<0.5 \text { if } N_{\mathrm{ch}}<50 \\
\end{array}$ & $\begin{array}{l}\text { constancy of light output along } \\
\text { the track, requirement is } \\
\text { tightened for low multiplicities }\end{array}$ \\
\hline & $\left|\Theta_{L F}-\Theta_{L R}\right|$ & $<10^{\circ}$ if $N_{\mathrm{ch}}<150$ & consistency of $L F$ and $L R$ \\
\hline
\end{tabular}

criteria are therefore tightened if the channel multiplicity $\left(N_{\mathrm{ch}}\right)$ observed in the event is small.

\subsection{Muon Detection Rates}

We compare the detector response as well as the event selection efficiency for all types of events: CR muons, muons induced by atmospheric neutrinos and muons from cosmic neutrinos with a hard energy spectrum, following an $E^{-2}$ power law. The numbers of triggered and selected events at each level, normalized to one year of data taking, are listed in table 2. With a flux of 
Table 2

Passing rates for signal and background Monte Carlo events. The signal expectation corresponds to a source flux of $E_{\nu}^{2} \times d N_{\nu} / d E_{\nu}=10^{-7} \mathrm{~cm}^{-2} \mathrm{~s}^{-1} \mathrm{sr}^{-1} \mathrm{GeV}$. The expectation for atmospheric neutrino events is listed separately for the "conventional" component and the "prompt" component (following [36](TIG) and [35](rqpm)). The fraction of prompt charm events with respect to the whole atmospheric neutrino sample is given in parentheses. The numbers of cosmic ray muon background events are shown separately for events that contain muon(s) from only one air shower (Atm $\left.\mu^{\text {single }}\right)$ and those that contain muons from two accidentally coinciding air showers (Atm $\left.\mu^{\text {double }}\right)$. The errors are statistical only.

\begin{tabular}{crrrrrr}
\hline & Trigger & Level 1 & Level 2 & \\
\hline \hline Cosmic $\nu$ & $3331 \pm 6$ & & $2172 \pm 4$ & $1089 \pm 3$ & \\
\hline Atm $\nu$ & $(824 \pm 4)$ & $\times 10^{3}$ & $(264 \pm 2)$ & $\times 10^{3}$ & $(91 \pm 1)$ & $\times 10^{3}$ \\
TIG & $(0.97 \pm 0.003)$ & $\times 10^{3}$ & $(0.40 \pm 0.002)$ & $\times 10^{3}$ & $(0.17 \pm 0.001)$ & $\times 10^{3}$ \\
& $(0.1 \%)$ & & $(0.2 \%)$ & & $(0.2 \%)$ & \\
& & & & & \\
rqpm & $(24.8 \pm 0.07)$ & $\times 10^{3}$ & $(11.08 \pm 0.04)$ & $\times 10^{3}$ & $(4.85 \pm 0.03)$ & $\times 10^{3}$ \\
& $(3 \%)$ & & $(4 \%)$ & & $(5 \%)$ & \\
\hline Atm $\mu^{\text {single }}$ & $(5.2 \pm 0.01)$ & $\times 10^{10}$ & $(1.3 \pm 0.01)$ & $\times 10^{9}$ & $(72 \pm 3)$ & $\times 10^{3}$ \\
Atm $\mu$ double & $(1.6 \pm 0.02)$ & $\times 10^{9}$ & $(4.6 \pm 0.3)$ & $\times 10^{7}$ & $(28 \pm 7)$ & $\times 10^{3}$ \\
\hline
\end{tabular}

$E_{\nu}^{2} \times d N_{\nu} / d E_{\nu}=10^{-7} \mathrm{~cm}^{-2} \mathrm{~s}^{-1} \mathrm{sr}^{-1} \mathrm{GeV}$ adopted as a benchmark for the flux of cosmic neutrinos, we expect more than 1000 signal events at level 2. At this stage, both the background from atmospheric neutrinos and the background from CR muons yield roughly $10^{5}$ events per year. The rqpm model for atmospheric charm predicts a contribution of almost 5000 prompt charm events to the atmospheric background. The TIG model predicts thirty times fewer events.

Figure 2 shows the zenith angle distribution for the reconstructed zenith angle $\Theta_{L R}$ of the four different event classes (Cosmic $\nu$, Atm $\nu$, Atm $\mu^{\text {single }}$ and Atm $\left.\mu^{\text {double }}\right)$ at different cut levels. The level 1 selection removes the bulk of low energy down-going CR-induced background. The angular cut on the zenith angles of the "first-guess" methods is still soft, so that most of the remaining background is located in the region around $30^{\circ}$ above the horizon. Level 2 then restricts the allowed zenith region to $5^{\circ}$ above the horizon, except for very bright, high multiplicity events. The remaining ordinary CR muon background (Atm $\mu^{\text {single }}$ ) at level 2 is then concentrated at the horizon and could be rejected with a tightened cut on the zenith angle, while the sample of CR-induced background composed of two air-showers (Atm $\mu^{\text {double }}$ ) still contains misreconstructed events that "fake" an upward track. However, filter level 2 does not contain a definite energy selection yet, required to separate the high energy signal of cosmic neutrinos from the atmospheric neutrino background. In the simplest approach this energy selection is an additional tight cut on the channel multiplicity. This final cut has to be optimized for 

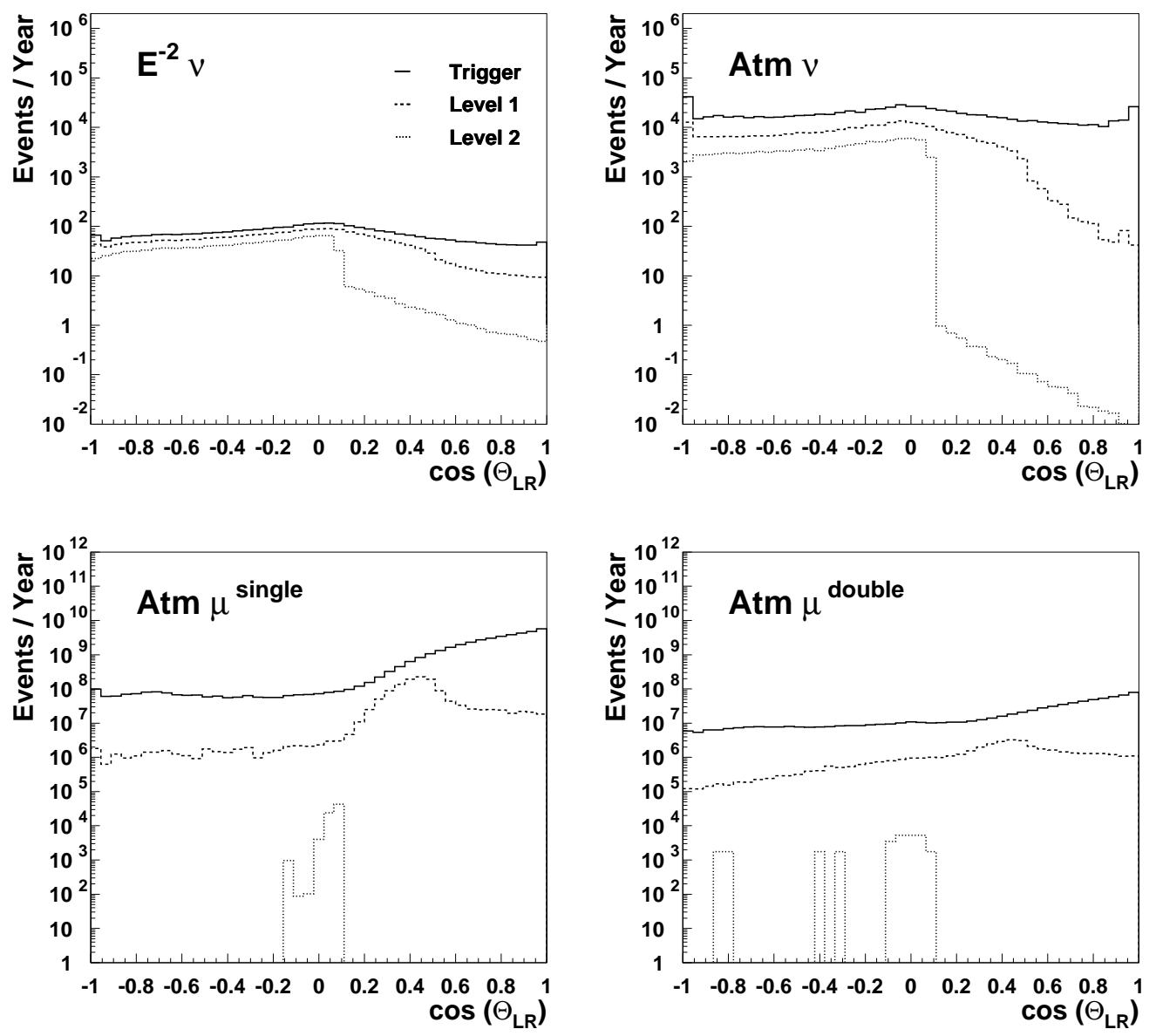

Fig. 2. Distribution of the reconstructed zenith angle for different event classes: Signal from a diffuse flux of cosmic neutrinos following an $E^{-2}$ spectrum (top left), atmospheric neutrino background including charm according to [35] (top right), atmospheric muon background from one single air-shower (bottom left) and two coincident air-showers (bottom right). The individual histograms in each plot correspond to: Trigger level (full lines), samples after applying level 1 and level 2 cuts (dashed and dotted lines, respectively). Event numbers are normalized to one year.

different analysis purposes (see section 5.2), but in any case it will lead to a drastic reduction of all three classes of background. In this analysis none of the CR muon events did pass this additional cut.

The energy spectra of muons generated by cosmic and atmospheric neutrinos are shown in figure 3. At the point of their closest approach to the detector center, muons from a cosmic $E^{-2}$ neutrino source typically have energies in the $\mathrm{TeV}-\mathrm{PeV}$ region, whereas the background of muons induced by atmospheric neutrinos peaks between 100 and $300 \mathrm{GeV}$. Figure 4 shows the channel multiplicity distributions of all event classes at level 2 . The signal of high energy cosmic neutrinos shows a clear excess at high multiplicities compared to the lower energy background events. 

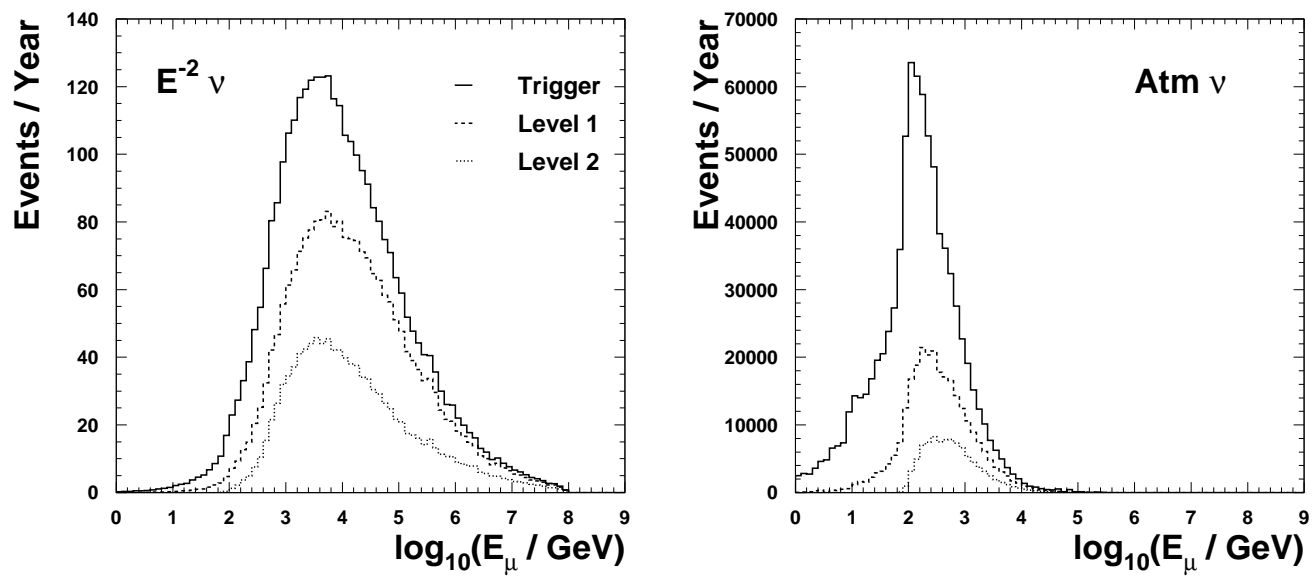

Fig. 3. Energy spectra of neutrino induced muons at different cut-levels: Signal from a $E^{-2}$-source (left), atmospheric neutrino background (right). The individual histograms in each plot correspond to: Trigger level (full lines), samples after applying level 1 cuts (dashed lines) and level 2 cuts (dotted lines). The energy spectra show the energy at the point of closest approach to the detector center.

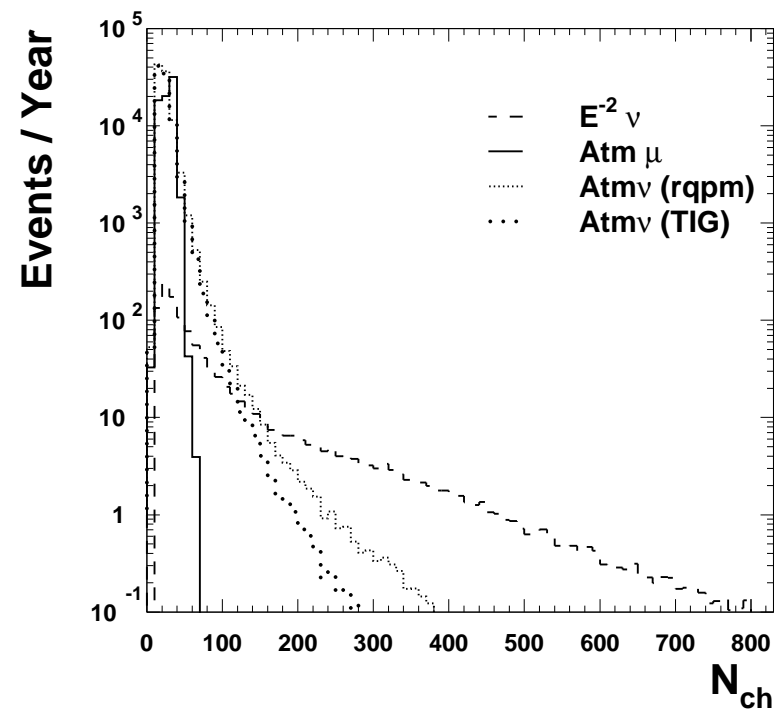

Fig. 4. Channel multiplicity for different event classes at level 2: For signal from $E^{-2}$ source (dashed), atmospheric neutrinos including the two alternative charm contributions TIG (sparse dots) and rqpm (dense dots) and CR muon events (full lines). 

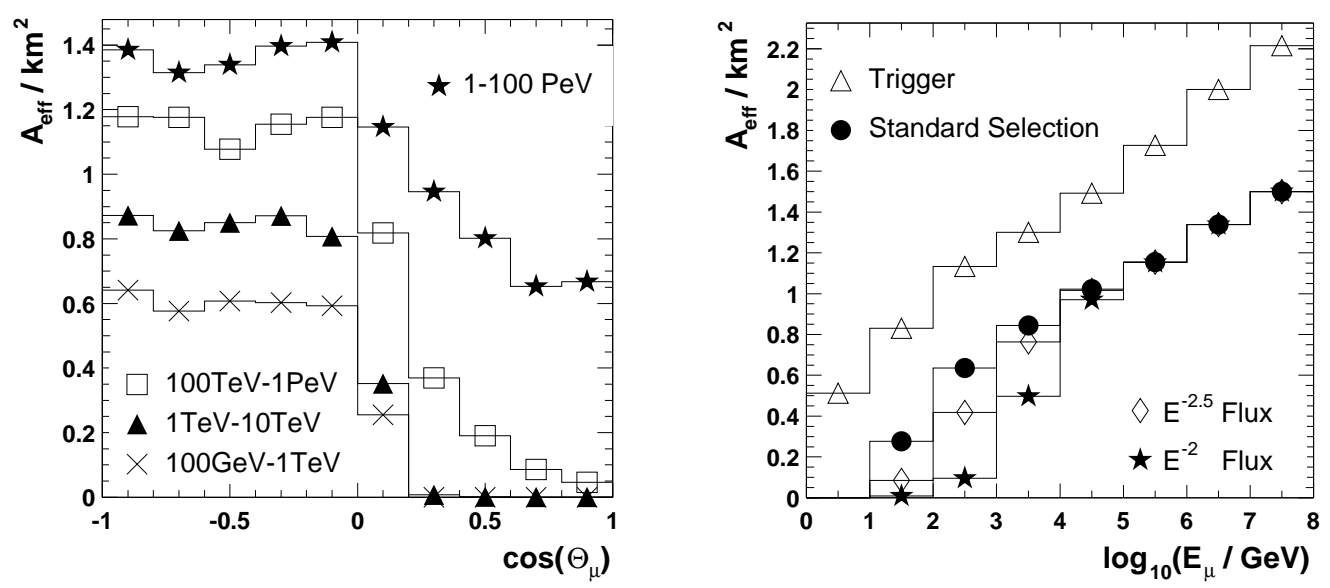

Fig. 5. Effective area as a function of the zenith angle after applying level 2 cuts (left) and as a function of the muon energy for different cut levels as described in the text (right). The zenith dependence is shown separately for different intervals of $E_{\mu}$. The energy dependence holds for muons for which $\cos \Theta_{\mu}<0$, i.e. muons that arrive from the northern sky. Energy cuts adjusted to signal spectra following $E^{-2}$ and $E^{-2.5}$ power laws change the energy threshold.

\subsection{Effective Detector Area}

As a measure of the detector efficiency we use the effective detector area, defined as

$$
A_{\text {eff }}\left(E_{\mu}, \Theta_{\mu}\right)=\frac{N_{\text {detected }}\left(E_{\mu}, \Theta_{\mu}\right)}{N_{\text {generated }}\left(E_{\mu}, \Theta_{\mu}\right)} \times A_{\text {gen }},
$$

where $N_{\text {generated }}$ is the number of muons in the test sample that have an energy $E_{\mu}$ at a given point within the fiducial volume and an incident zenith angle $\Theta_{\mu}$. In the following we give $E_{\mu}$ at the point of closest approach to the detector center (which might lie outside the geometrical detector volume). $N_{\text {detected }}$ is the number of events that trigger the detector or pass the cut level under consideration. The fraction of generated to triggered or selected events is scaled with the size of the generation plane $A_{\text {gen }}$, which is the cross-sectional area of the cylinder that contains all generated muon tracks with directions parallel to its axis.

The left plot in figure 5 shows the effective area at cut level 2 as a function of the zenith angle of the muon tracks. Results are shown for four separate energy intervals. The detector will have an effective detection area of one square kilometer for upward moving muons in the TeV range. Above $100 \mathrm{TeV}$ the selection allows for a detection of downgoing neutrinos, i.e. for an observation of the southern hemisphere $\left(\cos \theta_{\mu}>0\right)$. In the $\mathrm{PeV}$ range the effective area for downgoing muons is above $0.6 \mathrm{~km}^{2}$, increasing towards the horizon. This 
means that IceCube can observe a large part of our Galaxy, including the Galactic center.

The right plot in figure 5 shows the effective area as a function of the muon energy at closest approach to the detector center. Here, the effective area was calculated using a sample of muons which arrive from the lower hemisphere, i.e. using tracks with incident zenith angles larger than $90^{\circ}$ (or $\cos \theta_{\mu}<0$, accordingly). In that sense the numbers indicate average values for the northern

hemisphere. At trigger level the detector shows a sizeable acceptance even for low energy events.

The effective trigger area reaches one square kilometer at a few hundred GeV. Roughly $50 \%$ of all triggered events pass the "standard selection" (level2), independent of the muon energy. Best sensitivities to extraterrestrial neutrinos are obtained by increasing the energy threshold by additional cuts on the muon energy. The optimal threshold, i.e. the threshold where optimal sensitivity to one particular signal is achieved, is determined by the signal shape. For instance, a hard signal spectrum like $E^{-2}$ would suggest a tighter cut than a softer spectrum falling like $E^{-2.5}$. In order to show this effect the effective area was calculated after applying different energy cuts. In addition to trigger level and level 2, figure 5 shows the effective area after applying additional energy cuts optimized for probing hypothetical point sources with signal spectra following $E^{-2}$ and $E^{-2.5}$ (see section 5.3).

\subsection{Angular resolution}

The angular resolution of the detector is an important quantity for the search for neutrinos from point sources. A good angular resolution allows for a small search bin, resulting in a low background rate per bin. We characterize the angular resolution by the median of the distribution of the space angles between the true and reconstructed directions of the simulated muon tracks.

The angular resolution after applying level 2 cuts is shown in figure 6 as a function of the zenith angle of the muon tracks. In the energy region from $100 \mathrm{GeV}$ to $1 \mathrm{TeV}$ the pointing resolution approaches $1^{\circ}$ for tracks with zenith angles smaller than $140^{\circ}$. For nearly vertical tracks of low energy muons the angular resolution is worse, because these events are likely to cause hits in optical modules of only a single string. However, the reconstruction accuracy in this energy range is similar to the mean angle between the muon and the initial neutrino. In the most promising energy range, the region at few $\mathrm{TeV}$ and above, the resolution is substantially improved. Also, the zenith angle dependency of the pointing resolution weakens towards higher energies. Most of the signal in the $\mathrm{TeV}-\mathrm{PeV}$ region will be reconstructed with an accuracy 
significantly better than $1^{\circ}$. Current reconstruction methods achieve a resolution close to $0.5^{\circ}$ for events near the horizon. However, we expect significant improvement of the pointing resolution with further development of the reconstruction, in particular from including amplitude and waveform information. 


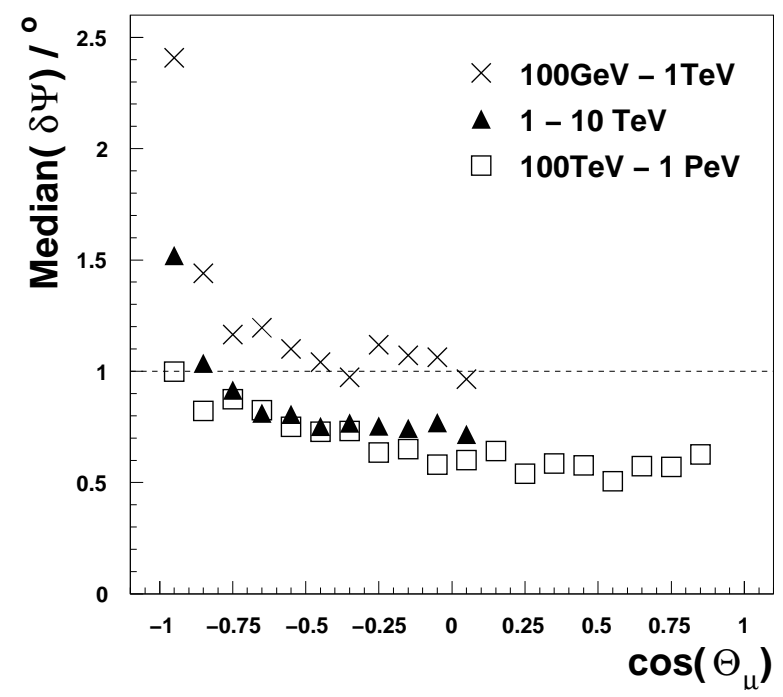

Fig. 6. Pointing resolution for neutrino-induced muon events. Shown is the median space angle error of the likelihood reconstruction as a function of the zenith angle of the incident track. The median was calculated for an energy spectrum $\propto E^{-2}$ and after applying level 2 cuts.

\section{Sensitivity to Astrophysical Sources of Muon Neutrinos}

In most theoretical models, the production of high energy CR is accompanied by the production of mesons. Prominent candidates for CR sources are putative cosmic accelerators like active galactic nuclei (AGN), microquasars, supernova remnants and gamma ray bursts (GRBs). Theoretical models for such objects usually involve shock acceleration of protons. The protons interact with ambient matter or radiation fields producing mesons that subsequently decay into neutrinos. The spectral distribution of neutrinos expected from cosmic accelerators is $d N_{\nu} / d E_{\nu} \propto E_{\nu}^{-2}$, or even harder, depending on the predominant meson production mechanism in the source and on full particulars of the acceleration.

The sum of all cosmic accelerators in the universe should produce an isotropic flux of high energy neutrinos, which would be observable as an excess above the diffuse flux of atmospheric neutrinos. The absolute flux of individual sources may be small, and requires a careful selection, if one wants to resolve it. However, in this case, one can strongly suppress background, because the number of background events will be reduced with the size of the spatial search bin or - in case of transient phenomena - the duration of the observation time window. 
In the following we calculate the sensitivity for diffuse fluxes of cosmic muon neutrinos as well as for fluxes from individual point sources, both steady and transient (GRBs). In contrast to former analyses, which were based on simple assumptions on the detector effective area as well as on its energy resolution $[22,23,24]$, the method we apply involves exclusively event observables that will be available from real data taken by IceCube.

\subsection{Calculation of the Sensitivity}

We explore the sensitivity of the IceCube detector to cosmic neutrino fluxes in two ways. First we consider the limits that would be placed on models of neutrino production if no events were to be seen above those expected from atmospheric neutrinos. Second, we evaluate the level of source flux required to observe an excess at a given significance level.

\subsubsection{Limit setting potential}

Feldman and Cousins have proposed a method to quantify the "sensitivity" of an experiment independently of experimental data by calculating the average upper limit $\bar{\mu}$ that would be obtained in absence of a signal [45]. It is calculated from the mean number of expected background events, $\left\langle n_{b}\right\rangle$, by averaging over all limits obtained from all possible experimental outcomes. The average upper limit is the maximum number of events that can be excluded at a given confidence level. That is, the experiment can be expected to constrain any hypothetical signal that predicts at least $\left\langle n_{s}\right\rangle=\bar{\mu}$ signal events.

From the $90 \%$ c.l. average upper limit, we define the "model rejection factor" (mrf) for an arbitrary source spectrum $\Phi_{s}$ predicting $\left\langle n_{s}\right\rangle$ signal events, as the ratio of the average upper limit to the expected signal [24]. The average flux limit $\Phi_{90}$ is found by scaling the normalization of the flux model $\Phi_{s}$ such that the number of expected events equals the average upper limit

$$
\Phi_{90}=\Phi_{s} \times \frac{\bar{\mu}_{90}}{\left\langle n_{s}\right\rangle} \equiv \Phi_{s} \times m r f
$$

\subsubsection{Discovery potential}

For our purposes, a phenomenon is considered "discovered" when a measurement yields an excess of 5-sigma over background, meaning that the probability of the observation being due to an upward fluctuation of background is less than $2.85 \times 10^{-7}$, being the the integral of the one-sided tail beyond 
5 -sigma of a normalized Gaussian. From the background expectation $\left\langle n_{b}\right\rangle$, we can determine the minimum number of events $n_{0}$ to be observed to produce the required significance as

$$
\sum_{n_{\mathrm{obs}}=n_{0}}^{\infty} P\left(n_{\mathrm{obs}} \mid\left\langle n_{b}\right\rangle\right) \leq 2.85 \cdot 10^{-7},
$$

where $P\left(n_{\text {obs }} \mid\left\langle n_{b}\right\rangle\right)$ is the Poisson probability for observing $n_{\text {obs }}$ background events. The minimum detectable flux $\Phi_{5 \sigma}$ for any source model can then be found by scaling the model flux $\Phi_{s}$ such that $\left\langle n_{s}\right\rangle+\left\langle n_{b}\right\rangle=n_{0}$.

If a real signal source of average strength $\Phi_{5 \sigma}$ is present, the probability of the combination of signal and background producing an observation sufficient to give the required significance (i.e. an observation of $n_{0}$ events or greater) is

$$
P_{5 \sigma}=\sum_{n_{\mathrm{obs}}=n_{0}}^{\infty} P\left(n_{\mathrm{obs}} \mid\left\langle n_{s}\right\rangle+\left\langle n_{b}\right\rangle\right)
$$

Thus we cannot say that an underlying signal strength will always produce an observation with 5 -sigma significance, but we can find the signal strength such that the probability of $P_{5 \sigma}$ is close to certainty e.g. $70 \%, 90 \%$ or $99 \%$.

\subsection{Diffuse Flux Sensitivity}

Many models have been developed that predict a diffuse neutrino flux to be expected from the sum of all active galaxies in the universe. First we will consider the potential of IceCube to both place a limit on, and detect, a generic diffuse flux following an $E^{-2}$ spectrum. After looking in detail at this case we summarize the capabilities of the detector to place limits on a few participating models with spectral shapes different from $E^{-2}$.

We use the simplest observable, the multiplicity $N_{\mathrm{ch}}$ of hit channels per event as an energy separation cut, in order to reject the steep spectrum of events induced by atmospheric neutrinos, and retain the events from the harder extraterrestrial diffuse spectrum. ${ }^{2}$ The correlation between the muon energy at closest approach to the detector center and channel multiplicity is shown in the left plot of figure 7 . The right plot shows the $N_{\text {ch }}$ distributions for an $E^{-2}$ signal compared to the atmospheric background.

$\overline{2}$ An improved energy separation is expected from the use of a more sophisticated energy reconstruction using individual hit amplitude and/or the full waveform information 

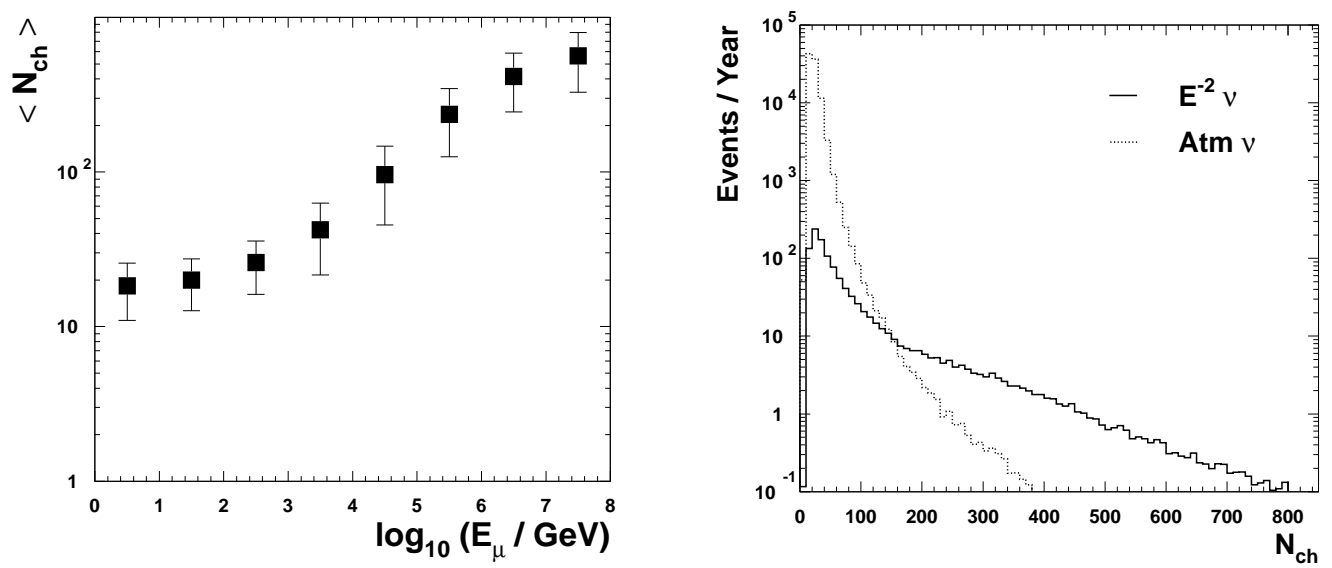

Fig. 7. Channel multiplicity. Left: Correlation between muon energy at closest approach to the detector center and detected channel multiplicity. Data points show to the mean number of OMs with a signal, averaged over one decade in energy. Error bars indicate the spread of the corresponding distribution. Right: Distribution of detected channel multiplicity for $E^{-2}$ signal and atmospheric background.
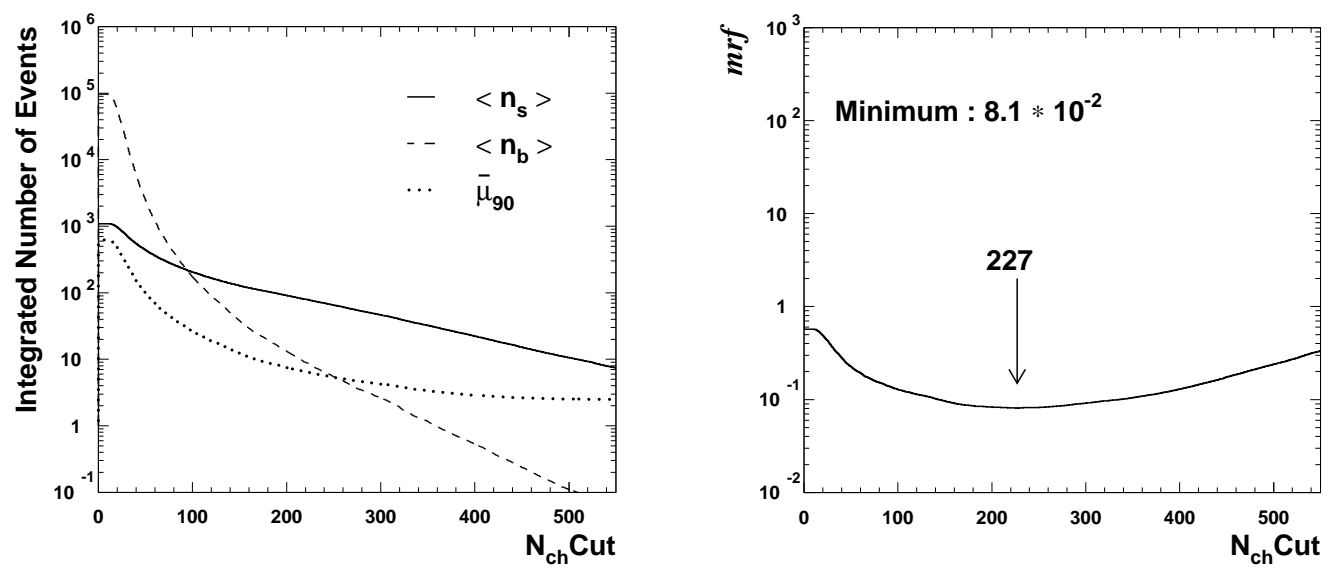

Fig. 8. Cut optimization.Left: Integrated distribution of detected channel multiplicity per event. Right: Model rejection factor with respect to a model source flux of $E_{\nu}^{2} \times d N_{\nu} / d E_{\nu}=10^{-7} \mathrm{~cm}^{-2} \mathrm{~s}^{-1} \mathrm{sr}^{-1} \mathrm{GeV}$ as a function of the applied $N_{\mathrm{ch}}$-cut.

In order to determine the $N_{\mathrm{ch}}$-cut that achieves the best sensitivity we optimize the cut with respect to the model rejection factor $(m r f)$ [24]. For each possible cut value we compute the mrf from the number of remaining signal and background events. The cut is placed, where the $m r f$ is minimized.

This procedure is illustrated in figure 8: The left plot shows the integrated multiplicity distribution for signal and background, together with the average 

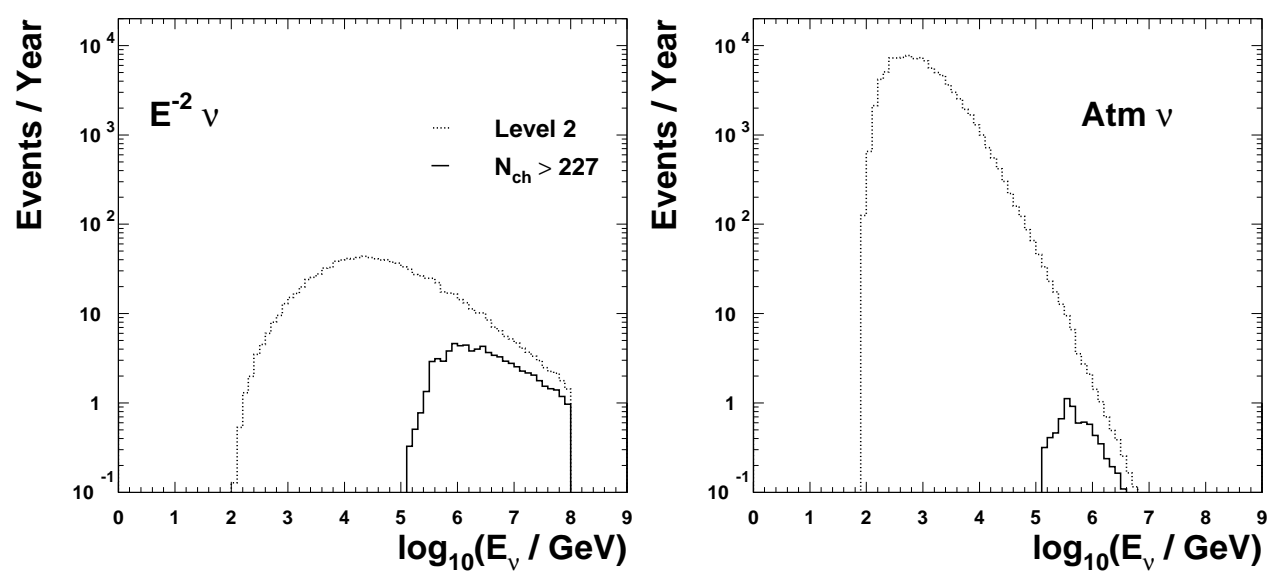

Fig. 9. Energy spectra of selected neutrinos for a $E^{-2}$ source (left) and atmospheric neutrinos (right). The selection is given by level 2 cuts (dotted lines) and application of the optimized cut $N_{\mathrm{ch}}>227$ (full lines). The cutoff in the signal spectrum at $10^{8} \mathrm{GeV}$ is due to the limited energy range of simulation.

upper limit $\bar{\mu}_{90}$. The right plot shows the $m r f$ as a function of the $N_{\mathrm{ch}}$-cut. The $m r f$ reaches its minimum of $8.1 \times 10^{-2}$ at $N_{\mathrm{ch}}=227$, corresponding to an overall flux limit of $E_{\nu}^{2} \times d N_{\nu} / d E_{\nu}=8.1 \times 10^{-9} \mathrm{~cm}^{-2} \mathrm{~s}^{-1} \mathrm{sr}^{-1} \mathrm{GeV}$. This flux limit applies for the flux of extraterrestrial muon-neutrinos to be measured at the Earth. In the presence of neutrino oscillations, the constraint on the flux escaping cosmic sources must be modified accordingly: For maximal mixing $[46,47]$ between muon- and tau-neutrinos during propagation to the Earth, one would expect the flux of muon neutrinos at the Earth to be half as large as the flux at the source. So the limit on the muon neutrino flux produced in cosmic sources is higher by a factor of two. In the following "cosmic neutrino flux" refers to the intensity of muon neutrinos measured at the Earth.

The simulated cosmic neutrino flux of $E_{\nu}^{2} \times d N_{\nu} / d E_{\nu}=1 \times 10^{-7} \mathrm{~cm}^{-2} \mathrm{~s}^{-1} \mathrm{sr}^{-1} \mathrm{GeV}$ yields 74 signal events passing the optimized cut, compared to 8 background events from atmospheric neutrinos. The background expectation was calculated using the rqpm model for the prompt charm contribution, according to which prompt charm decays account for $80 \%$ of the remaining atmospheric neutrinos. The prediction according to the TIG model would result in an improvement of the average flux limit by roughly a factor of two.

The energy spectra of the incident signal and background neutrinos are shown in figure 9. The final cut, as it is placed here, results in a detection threshold of about $100 \mathrm{TeV}$. This threshold is the result of an optimization to one particular signal hypothesis, an $E^{-2}$ neutrino spectrum extending up to energies of $10^{8} \mathrm{GeV}$. The artificial cutoff in the signal spectrum at $10^{8} \mathrm{GeV}$ (where the simulation ends) neglects additional events above this energy. Without the artifical cutoff one would get a slightly improved limit. 


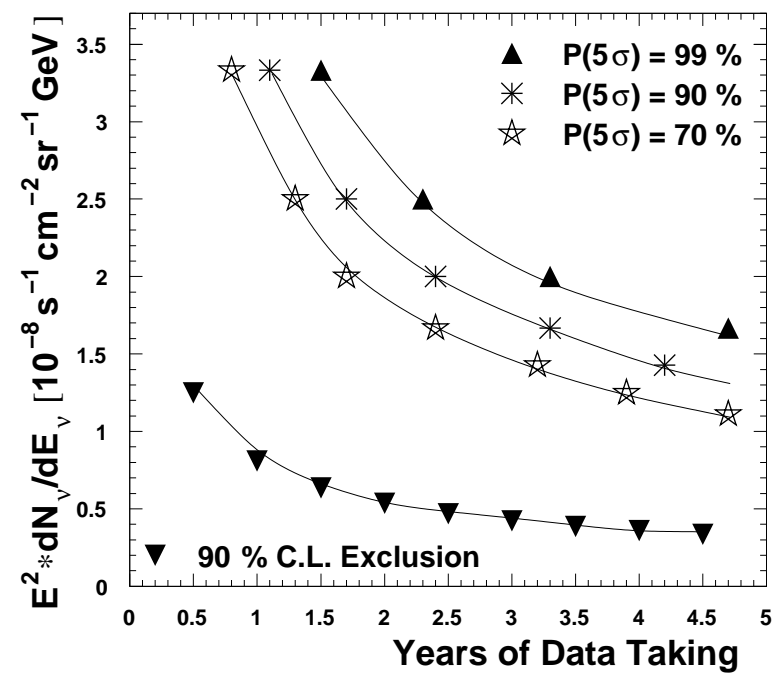

Fig. 10. Sensitivity to diffuse neutrino fluxes: Improvement with time.

The sensitivity obtained after one year of data taking is already well below the diffuse bound calculated by Waxman and Bahcall [48]. (This limit holds for optically thin CR sources, under the assumption that these sources produce the observed flux of high energy CR.) A flux at the level $E_{\nu}^{2} \times d N_{\nu} / d E_{\nu}=2.4 \times$ $10^{-8} \mathrm{~cm}^{-2} \mathrm{~s}^{-1} \mathrm{sr}^{-1} \mathrm{GeV}$ is needed for a 5-sigma observation after a period of one year. This flux is 40 times below the present best established $90 \%$ c.l. upper limit [9]. The improvement with time of the exclusion and discovery potential of the detector is summarized in table 3. As the exposure time increases, the optimal multiplicity cut becomes tighter, resulting in a better separation of signal and background. After data is taken over five years, the sensitivity is improved by a factor of about 2.5. The 5-sigma detection level given in table 3 corresponds to the flux, for which the event rate from signal plus background exceeds the 5 -sigma threshold. The signal strength at which the 5 -sigma excess is produced at a fixed probability, is shown in figure 10 as a function of time. A signal of $E_{\nu}^{2} \times d E_{\nu} / d N_{\nu}=1 \times 10^{-8} \mathrm{~cm}^{-2} \mathrm{~s}^{-1} \mathrm{sr}^{-1} \mathrm{GeV}$, for instance, would be detected with a probability of $70 \%$ after five years of datataking.

Apart from the generic case of an $E^{-2}$ spectrum, which is typical for scenarios that involve meson production in interactions of shock accelerated $\mathrm{CR}$ with matter, we have varied the signal slope towards flatter spectra. Such spectra would be expected from environments where CR predominantly interact on photon fields, e.g. AGN jets [49]. The average differential limits for an assumed observation period of three years are listed in table 4 .

Mannheim, Protheroe and Rachen have calculated an upper bound on the diffuse neutrino flux arising from photo-hadronic interactions in unresolved AGN jets in the universe. Their flux bound is shown in figure 11 labeled 
Table 3

Sensitivity to diffuse neutrino fluxes. Expected limits and minimal detectable fluxes in units of $\mathrm{cm}^{-2} \mathrm{~s}^{-1} \mathrm{sr}^{-1} \mathrm{GeV}$ for a generic $E^{-2}$ source spectrum. Event numbers correspond to a hypothetical source strength of $E_{\nu}^{2} \times d N_{\nu} / d E_{\nu}=1 \times$ $10^{-7} \mathrm{~cm}^{-2} \mathrm{~s}^{-1} \mathrm{sr}^{-1} \mathrm{GeV}$.

\begin{tabular}{lcccccc}
\hline years & $N_{\text {ch }}$ Cut & $\left\langle n_{s}\right\rangle$ & $\left\langle n_{b}\right\rangle$ & $\bar{\mu}_{90}$ & $E^{2} \frac{d N}{d E}(90 \%$ c.l. $)$ & $E^{2} \frac{d N}{d E}(5 \sigma)$ \\
\hline \hline 1 & 227 & 76.4 & 8.0 & 6.1 & $8.1 \cdot 10^{-9}$ & $2.6 \cdot 10^{-8}$ \\
3 & 244 & 204.8 & 18.4 & 8.7 & $4.2 \cdot 10^{-9}$ & $1.2 \cdot 10^{-8}$ \\
5 & 276 & 272.5 & 18.0 & 8.6 & $3.2 \cdot 10^{-9}$ & $9.9 \cdot 10^{-9}$ \\
\hline
\end{tabular}

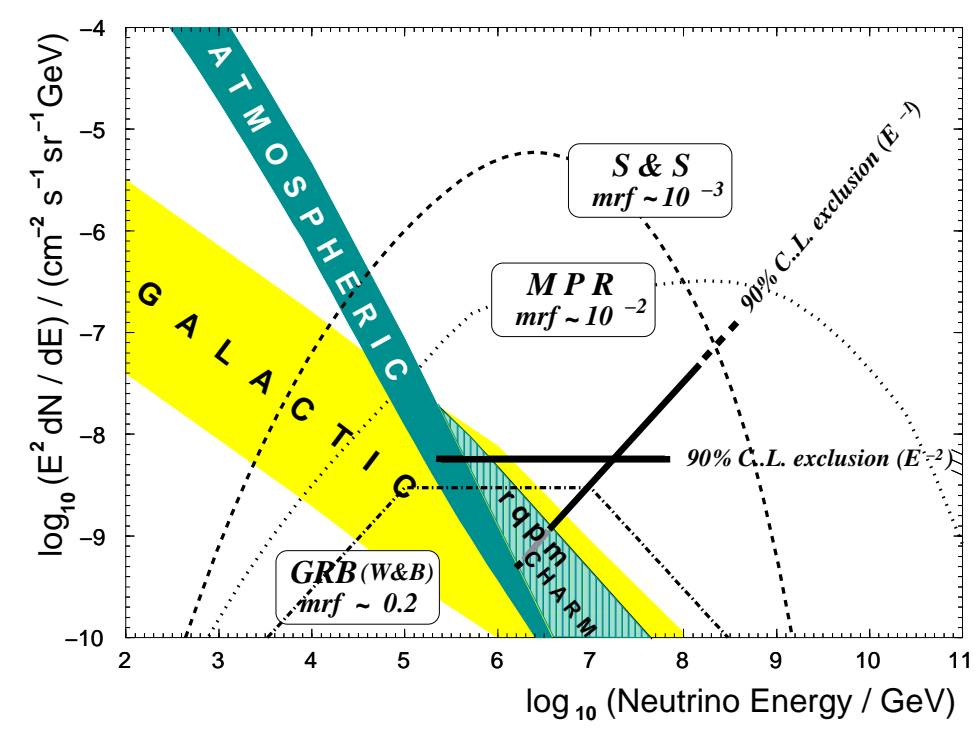

Fig. 11. Expected sensitivity of the IceCube detector. Solid lines indicate the $90 \%$ c.l. limit for various differential spectra, calculated for a data taking period of three years. The lines extend over the energy range containing $90 \%$ of the expected signal. The dashed line indicates the Stecker and Salamon model for photo-hadronic interactions in AGN cores [50]. The dotted line corresponds to the Mannheim, Protheroe and Rachen upper bound on the neutrino emission from photo-hadronic interactions in AGN jets [49]. Also shown is the GRB model by Waxman and Bahcall [48] (dash-dotted line). The $m r f$ was calculated exemplarily for an observation of 500 bursts (see section 5.4).

MPR. We have determined the model rejection potential for this particular shape (and for energies below $10^{8} \mathrm{GeV}$ ) to be $\operatorname{mrf}=1.9 \cdot 10^{-2}$, meaning that IceCube will be sensitive to fluxes of similar shape, but fifty times smaller than the $\boldsymbol{M P R}$ maximum model.

Finally, we have selected one particular model by Stecker and Salamon [50] for neutrinos from proton interactions on the UV thermal photon field in AGN 
Table 4

Sensitivity to diffuse neutrino fluxes of various shapes. Expected limits and minimum detectable fluxes in units of $\mathrm{cm}^{-2} \mathrm{~s}^{-1} \mathrm{sr}^{-1} \mathrm{GeV}$ for different signal hypotheses. Numbers correspond to an exposure time of three years.

\begin{tabular}{lccc}
\hline Source Model & $N_{\text {ch }}$ Cut & $\bar{\mu}_{90}$ & Flux Limit $(=\Phi \times m r f)$ \\
\hline \hline$E^{-1}$ & 427 & 3.3 & $d N_{\nu} / d E_{\nu}=3.1 \cdot 10^{-16}(E / \mathrm{GeV})^{-1}$ \\
$E^{-1.5}$ & 336 & 4.9 & $d N_{\nu} / d E_{\nu}=1.5 \cdot 10^{-12}(E / \mathrm{GeV})^{-1.5}$ \\
\hline \hline SS96 & 250 & 8.3 & $\Phi_{\mathrm{SS} 96} \times 2.3 \cdot 10^{-3}$ \\
MPR & 324 & 5.2 & $\Phi_{\mathrm{MPR}} \times 1.9 \cdot 10^{-2}$ \\
\hline
\end{tabular}

cores. The corresponding diffuse flux prediction is labeled $\boldsymbol{S} \& \boldsymbol{S}$ in figure 11. The model rejection factor in this case is $m r f=2.3 \cdot 10^{-3}$. Figure 11 also shows the $90 \%$ c.l. expected limits on an $E^{-2}\left(E^{-1}\right)$ neutrino flux.

\subsection{Sensitivity to Point Sources}

An excess of events from a particular direction in the sky suggests the existence of a point source. The ability of the detector to reconstruct muon tracks to within $1^{\circ}$ of their true direction allows a search window to be used that greatly reduces the background, while retaining a large fraction of the signal. This allows for a loosening of the energy separation cut.

We restrict this analysis to the case of a point source search for candidate sources in the northern sky. That is, we do not simulate a cluster or grid search, but we consider the case where an angular search bin is fixed by the direction of the candidate source under test. In reality the sensitivity will depend on the declination of the source location. For simplicity of presentation we calculate averaged event rates for all declinations throughout the northern sky.

We use an angular search cone of $1^{\circ}$ centered about the direction of a hypothetical point source. After application of the standard cut selection, we optimize the $N_{\mathrm{ch}}$-cut with respect to the model rejection potential for a point source following an $E^{-2}$ spectrum. A cut at a channel multiplicity of $N_{\mathrm{ch}}=30$, combined with the angle cut of one degree, leads to the best average flux upper limit of $E_{\nu}^{2} \times d N_{\nu} / d E_{\nu}=5.5 \times 10^{-9} \mathrm{~cm}^{-2} \mathrm{~s}^{-1} \mathrm{GeV}$ after one year of data taking. A flux three times greater, will on average, produce a 5-sigma signal.

Table 5 and figure 12 summarize the improvement of the limit with increased exposure time. After three years of operation IceCube can be expected to place flux limits on potential sources at a level $E_{\nu}^{2} \times d N_{\nu} / d E_{\nu} \sim 2.4 \times$ $10^{-9} \mathrm{~cm}^{-2} \mathrm{~s}^{-1} \mathrm{GeV}$, while the discovery probability for a flux three times stronger 
Table 5

Sensitivity to point sources. Expected limits and minimal detectable fluxes in units of $\mathrm{cm}^{-2} \mathrm{~s}^{-1} \mathrm{GeV}$ for a generic $E^{-2}$ source spectrum and different exposure times. signal event rates correspond to a hypothetical source strength of $E_{\nu}^{2} \times d N_{\nu} / d E_{\nu}=1 \times 10^{-7} \mathrm{~cm}^{-2} \mathrm{~s}^{-1} \mathrm{GeV}$, background event rates include rqpm charm neutrinos.

\begin{tabular}{lcccccc}
\hline years & $N_{\mathrm{ch}}$ Cut & $\left\langle n_{s}\right\rangle$ & $\left\langle n_{b}\right\rangle$ & $\bar{\mu}_{90}$ & $E^{2} \frac{d N}{d E}(90 \%$ c.l. $)$ & $E^{2} \frac{d N}{d E}(5 \sigma)$ \\
\hline \hline 1 & 30 & 62.8 & 1.4 & 3.6 & $5.5 \cdot 10^{-9}$ & $1.7 \cdot 10^{-8}$ \\
3 & 40 & 142.3 & 1.3 & 3.5 & $2.4 \cdot 10^{-9}$ & $7.2 \cdot 10^{-9}$ \\
5 & 42 & 213.7 & 1.4 & 3.6 & $1.7 \cdot 10^{-9}$ & $4.9 \cdot 10^{-9}$ \\
\hline
\end{tabular}

is lager than $70 \%$. After five years of operation a source emitting a flux of $E_{\nu}^{2} \times d N_{\nu} / d E_{\nu} \sim 6 \times 10^{-9} \mathrm{~cm}^{-2} \mathrm{~s}^{-1} \mathrm{GeV}$ would be observed at 5-sigma significance with a probability of $70 \%$.

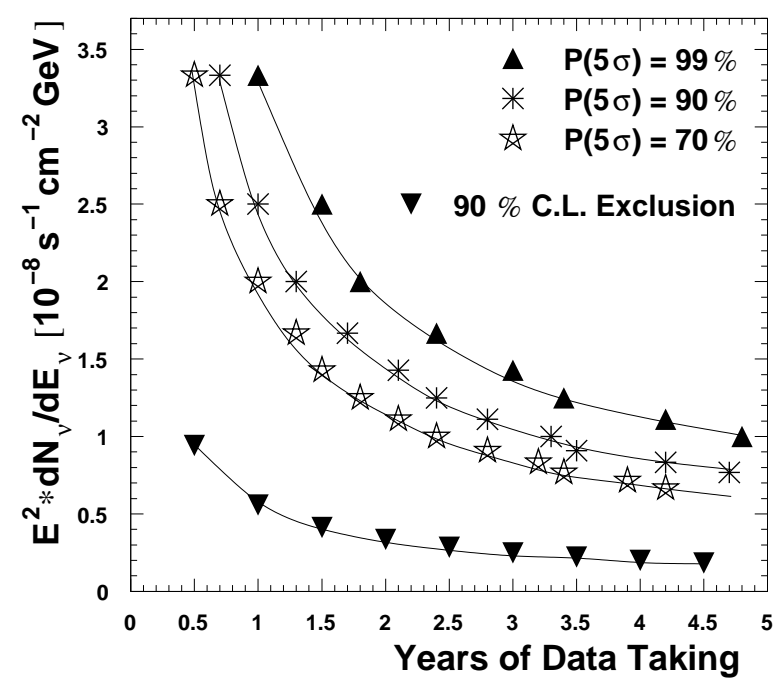

Fig. 12. Sensitivity to point-like neutrino emission: Improvement with time.

Figure 13 shows the energy spectra of both the remaining signal events and the remaining events from the atmospheric neutrino background after applying standard cuts and after cutting at $N_{\mathrm{ch}}>30$. This cut results in an effective energy threshold of $1 \mathrm{TeV}$. Since most of the signal is in the $\mathrm{TeV}$ region, the energy cut-off of the Monte Carlo simulation has negligible impact on the result. Above results are valid for the rqpm prediction for prompt neutrinos. Using the TIG model improves the sensitivity by about $2 \%$.

As for the diffuse signal, we have tested different exponential slopes for the signal hypothesis. The results listed in table 6 correspond to three years of 
Table 6

Sensitivity to point source fluxes of various exponential slopes. Expected limits and minimal detectable fluxes in units of $\mathrm{cm}^{-2} \mathrm{~s}^{-1} \mathrm{GeV}$ for different signal hypotheses. Numbers correspond to an exposure time of three years.

\begin{tabular}{lccl}
\hline Source Model & $N_{\text {ch }}$ Cut & $\bar{\mu}_{90}$ & Flux Limit $(=\Phi \times m r f)$ \\
\hline \hline$E^{-1}$ & 58 & 2.7 & $d N_{\nu} / d E_{\nu}=2.4 \cdot 10^{-15}(E / \mathrm{GeV})^{-1}$ \\
$E^{-1.5}$ & 49 & 2.9 & $d N_{\nu} / d E_{\nu}=4.5 \cdot 10^{-12}(E / \mathrm{GeV})^{-1.5}$ \\
$E^{-2}$ & 40 & 3.5 & $d N_{\nu} / d E_{\nu}=2.4 \cdot 10^{-9}(E / \mathrm{GeV})^{-2}$ \\
$E^{-2.5}$ & 24 & 6.1 & $d N_{\nu} / d E_{\nu}=3.8 \cdot 10^{-5}(E / \mathrm{GeV})^{-2.5}$ \\
\hline
\end{tabular}

data taking.
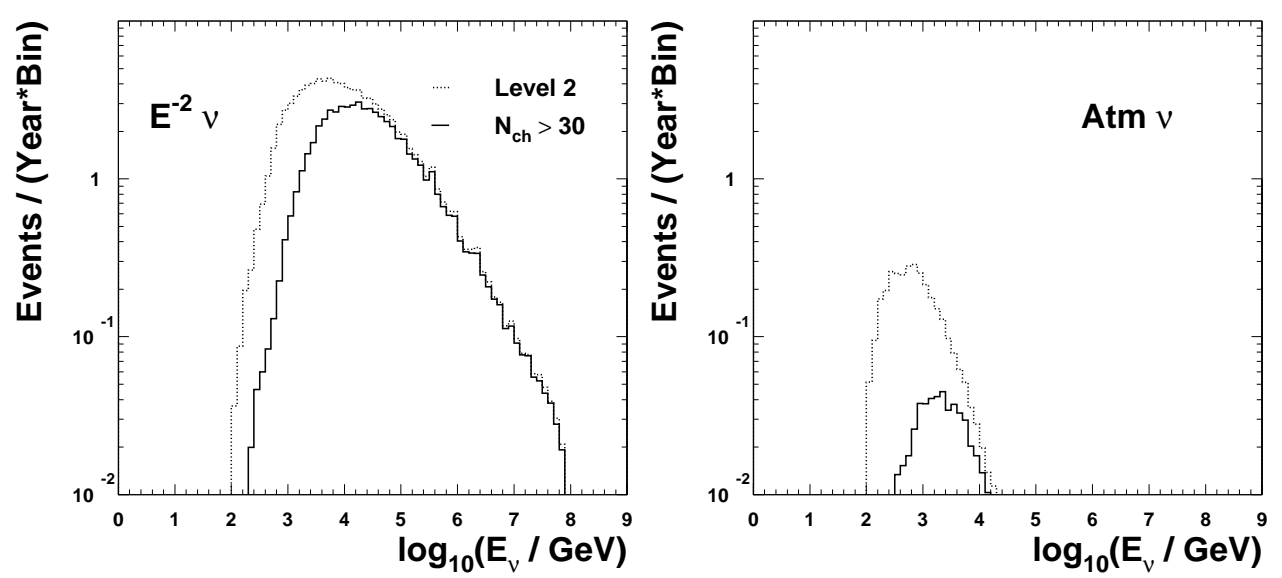

Fig. 13. Energy spectra of selected neutrinos for a $E^{-2}$ source (left) and atmospheric neutrinos including rqpm charm (right). The selection corresponds to level 2 cuts (dotted lines) and additional application of the optimized cut $N_{\mathrm{ch}}>30$ (full lines).

\subsection{Gamma Ray Burst Sensitivity}

Although the progenitors of GRBs are unknown, observations indicate the existence of a fireball. The coexistence of nucleons and photons in the fireball may result in the production of neutrinos.

Waxman and Bahcall [48] calculated the expected flux of neutrinos from the sum of all GRBs by assuming that they are the source of the observed flux of cosmic rays. The Waxman-Bahcall model results in a broken power-law 
neutrino spectrum given by

$$
\frac{d N_{\nu}}{d E_{\nu}}=\left\{\begin{array}{l}
\frac{A}{E_{\nu} E_{\nu}^{\mathrm{b} 1}}, \quad E_{\nu}<E_{\nu}^{\mathrm{b} 1} \\
\frac{A}{E_{\nu}^{2}} \quad, E_{\nu}^{\mathrm{b} 1}<E_{\nu}<E_{\nu}^{\mathrm{b} 2},
\end{array}\right.
$$

where the break energy $E_{\nu}^{\mathrm{b} 1}$ lies at $\sim 10^{5} \mathrm{GeV}$. Above $E_{\nu}^{\mathrm{b} 2}=10^{7} \mathrm{GeV}$ the spectrum steepens again by one power in energy. With a full sky GRB rate of $\sim 1000$ per year, as assumed by Waxman and Bahcall, the normalization constant in equation 5 would amount to $A \sim 3 \times 10^{-9} \mathrm{~cm}^{-2} \mathrm{~s}^{-1} \mathrm{sr}^{-1} \mathrm{GeV} .{ }^{3}$

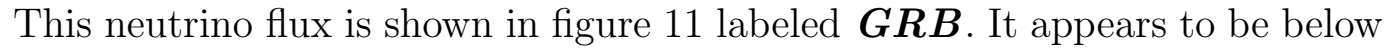
the diffuse flux sensitivity level of IceCube. However, the search for neutrinos accompanying a GRB is essentially background-free, because one looks for neutrino events that are coincident in direction and time to the satellite observation.

The search for neutrinos from GRBs involves summing over the observation time and spatial search windows for many separate bursts. For this analysis we have used a hypothetical observation duration of 10 seconds and a spatial search cone of $10^{\circ}$ centered about the direction of each GRB. We have only considered events in the northern sky, where we can be sure that the search will not be limited by downgoing CR muon background. From 500 bursts in $2 \pi$ sr we would expect 13 neutrino-induced up-going muons per year after applying standard level 2 quality cuts (table 1). The background of atmospheric neutrinos is strongly reduced by the spatial and temporal coincidence requirements. With almost full retention of the signal, the atmospheric neutrino background expectation is reduced to roughly 0.1 event. The smallness of the background expectation allows one to exclude signals of mean intensity $\left\langle n_{s}\right\rangle=\bar{\mu}_{90}=2.5$ events per year at $90 \%$ classical confidence, meaning that the experiment will be sensitive to a neutrino flux roughly five times smaller than the flux calculated by Waxman and Bahcall (equation 5). This also means that the observation of 100 bursts would suffice to exclude the Waxman and Bahcall model. A 5-sigma detection would require the observation of $n_{0}=5$ events, which corresponds to the mean number of events expected from 203 bursts. In this case the probability to actually observe a 5-sigma excess is about $58 \%$. With 500 bursts this probability climbs to $99 \%$. The time period after which we can expect a detection depends on the efficiency of the gamma ray observations, since the search strategy requires the GRBs to be triggered by satellites. Assuming that future gamma ray observations will provide a few hundred triggered burst per year, we can conclude that IceCube has excellent prospects to reveal the neutrino signal possibly emerging from GRBs within a very short time: The analysis of data taken over one year would presumably suffice to yield a 5-sigma signal (provided the model by Waxman and

3 A more recent calculation yielded a normalization constant which is about three times larger [51]. 
Bahcall predicts neutrino fluxes at the right scale). Moreover, the sensitivity given above is obtained when employing the most conservative search strategy, namely searching only one hemisphere for the signal of up-going neutrinos. However, one can be convinced that the drastic background reduction due to the small observation time window will result in a sizable acceptance also for downward signal.

\subsection{Systematic Uncertainties and Possible Improvements}

The present systematic uncertainty of the given flux limits is dominated by three components. The largest is the uncertainty in the angular dependence of the OM sensitivity, including the effect of the refrozen ice around the OM. A local increase in light scattering from air bubbles trapped in the vicinity of the OM translates into a modulation of its angle-dependent acceptance. This component is followed in size by uncertainties in the absolute OM sensitivity and in the optical properties of the bulk ice. For the comparatively small AMANDA-B10 array the inclusion of all components of uncertainty weakens the point source flux limit by $25 \%$ compared to standard simulation values [52]. The variation of some of these parameters in simulations of the larger AMANDA-II array and for IceCube indicates that for larger arrays the systematic uncertainties of the basic input parameters become less important, with the exception of muon energies close to the detection threshold. For instance, increasing the absolute OM sensitivity in IceCube by a factor of 2 results in a $25(10) \%$ larger effective area at 1 (10) TeV. Taking into account that uncertainties in limits depend more weakly than linearly on uncertainties in effective area [52], we estimate the overall uncertainties of the $E^{-2}$ limits derived above to be at most $20 \%$.

On the other hand, we may expect that improved detector properties for IceCube as compared to AMANDA will result in smaller systematic uncertainties and a better performance. First, the use of glass spheres and PMT glass with better UV transparency or, alternatively, a covering of the glass spheres with wavelength shifter, will enhance the OM sensitivity in the UV region and result in a better light collection. This will particularly increase the sensitivity and angular resolution at low energies. Information obtained from the full waveform will improve both the angular resolution and the energy reconstruction at high energies. Waveform information will be used in AMANDA from 2003 on, and methods to make efficient use of the corresponding information are under development. Finally, the information from the IceTop surface array will enhance the rejection power with respect to downward moving atmospheric muons. This method, unique to IceCube, is expected to be particularly helpful for muons from coincident, independent air showers and would allow a loosening other rejection criteria, thereby enhancing the signal efficiency. 


\section{Summary}

We have described the performance of the IceCube detector in searching for muons from extraterrestrial neutrinos in the TeV-PeV energy range.

A Monte Carlo simulation of a realistic model detector was used to assess the sensitivity of the experiment. We have simulated both neutrino-induced muons and muons produced from cosmic-ray interactions in the atmosphere with sufficient statistics to establish event selection criteria and infer event rates to be expected from each event class. The trigger rate due to down-going muons produced in the atmosphere was found to be $1.7 \mathrm{kHz}$ including a $50 \mathrm{~Hz}$ rate due to non-correlated air-showers that produce time-coincident muons within the detector. Muons induced by atmospheric neutrinos are expected to cause about 0.8 million triggers per year. A benchmark flux of $E_{\nu}^{2} \times d N_{\nu} / d E_{\nu}=$ $10^{-7} \mathrm{~cm}^{-2} \mathrm{~s}^{-1} \mathrm{sr}^{-1} \mathrm{GeV}$ for the diffuse signal of astrophysical neutrinos results in 3300 triggers per year. Roughly a third of them pass quality cuts which at the same time reduce the background rate from misreconstructed downward muon tracks to the level of well reconstructed upward muons from atmospheric neutrinos.

In order to quantify the detector acceptance, we have computed the effective detector area. After applying a set of standard quality criteria, the effective area exceeds one square kilometer for upward-going muons with energies of $10 \mathrm{TeV}$ and above. At this stage, $50 \%$ of all muons of this energy will be reconstructed with an accuracy of $0.8^{\circ}$ or better. For energies above $100 \mathrm{TeV}$, the angular acceptance with respect to well identified extraterrestrial neutrinos extends above the horizon and the effective area reaches $0.6 \mathrm{~km}^{2}$ for downgoing muons in the $\mathrm{PeV}$ range. This means that at high energies IceCube can observe a large part of the Galaxy, including the galactic center.

In order to quantify the sensitivity to fluxes of astrophysical neutrinos, we have determined the flux normalization for a generic $E^{-2}$ differential energy spectrum that correspond to a detection with 5-sigma significance, or, in absence of signal, a $90 \%$ c.l. limit. We found a diffuse source strength of $E_{\nu}^{2} \times d N_{\nu} / d E_{\nu}=10^{-8} \mathrm{~cm}^{-2} \mathrm{~s}^{-1} \mathrm{sr}^{-1} \mathrm{GeV}$ for the 5-sigma detection level and $4 \times 10^{-9} \mathrm{~cm}^{-2} \mathrm{~s}^{-1} \mathrm{sr}^{-1} \mathrm{GeV}$ for the exclusion potential of the detector, given an observation time of three years. This is two orders of magnitude below present experimental limits. For point-like neutrino emission we found that, after three years, a flux of $E_{\nu}^{2} \times d N_{\nu} / d E_{\nu}=7 \times 10^{-9} \mathrm{~cm}^{-2} \mathrm{~s}^{-1} \mathrm{GeV}$ would result in a 5-sigma excess over background, while a flux of $E_{\nu}^{2} \times d N_{\nu} / d E_{\nu}=$ $2 \times 10^{-9} \mathrm{~cm}^{-2} \mathrm{~s}^{-1} \mathrm{GeV}$ can be excluded at $90 \%$ c.l. Both numbers are averaged over all declinations throughout the northern sky. Integrated over all neutrino energies above $1 \mathrm{TeV}$, these fluxes transform to $F_{\nu}(>1 \mathrm{TeV})=7(2) \times 10^{-12}$ $\mathrm{cm}^{-2} \mathrm{~s}^{-1}$. 
We have also calculated the potential of IceCube to detect neutrinos in coincidence with gamma ray bursts, following the model of Waxman and Bahcall. We found that a 5-sigma signal is expected from the observation of about 200 bursts, while an observation of 100 bursts would suffice to rule out the Waxman and Bahcall model.

\section{Acknowledgements}

This research was supported by the following agencies: National Science FoundationOffice of Polar Programs, National Science Foundation-Physics Division, University of Wisconsin Alumni Research Foundation, USA; Swedish Research Council, Swedish Polar Research Secretariat, Knut and Alice Wallenberg Foundation, Sweden; German Ministry for Education and Research, Deutsche Forschungsgemeinschaft (DFG), Germany; Fund for Scientific Research (FNRS-FWO), Flanders Institute to encourage scientific and technological research in industry (IWT), Belgian Federal Office for Scientific, Technical and Cultural affairs (OSTC), Belgium; Inamori Sceience Foundation, Japan; FPVI, Venezuela; The Netherlands Organization for Scientific Research (NWO).

\section{References}

[1] T. K. Gaisser, F. Halzen and T. Stanev, Phys. Rep. 258 (1995) 173.

[2] J. G. Learned and K. Mannheim, Ann. Rev. Nucl. Part. Sci. 50 (2000) 679.

[3] C. Spiering, Progr. in Particle and Nucl. Phys. 48 (2002) 43.

[4] P. K. F. Grieder et al., Nucl. Phys. Proc. Suppl. 43 (1995) 145.

[5] V. A. Balkanov et al., Nucl. Phys. Proc. Suppl., 75A (1999) 409.

[6] E. Andres et al., Astropart. Phys. 13 (2000) 1.

[7] J. Ahrens et al., Phys. Rev. D 66 (2002) 012005.

[8] I. A. Belolaptikov et al., Phys. Atom. Nucl. 63 (2000) 951.

[9] J. Ahrens et al., submitted to Phys. Rev. Lett., astro-ph/0303218.

[10] T. Montaruli et al., Proc. 26th ICRC 2 (1999) 213.

[11] S. Matsuno, Proc. 27th ICRC 3 (2001) 1065.

[12] E. Aslanides et al., A Deep Sea Telescope for High Energy Neutrinos, Proposal (1999), astro-ph/9907432. 
[13] S. Bottai, Proc. 26th ICRC 2 (1999) 456.

[14] G. Riccobene, Proc. Workshop on Methodical Aspects of Underwater/Ice Neutrino Telescopes, DESY-PROC-2002-01 (2002) 61.

[15] J. Ahrens et al., The IceCube NSF Proposal (2000).

[16] A. Biron et al., Participation of DESY-Zeuthen in the IceCube Project, Proposal to the DESY PRC (2001).

[17] J. Ahrens et al., IceCube Conceptual Design Document (2001).

[18] A. Goldschmidt, Proc. 27th ICRC 3 (2001) 1237.

[19] M. Leuthold, Proc. Workshop on Simulation and Analysis Methods for Large Neutrino Telescopes, DESY-PROC-1999-01 (1999) 484.

[20] H. Wissing, Diploma Thesis, Humboldt Universität zu Berlin (2001).

[21] X. Bai et al., Proc. 27th ICRC 3 (2001) 981.

[22] I. F. M. Albuquerque, J. Lamoureux and G. Smoot, hep-ph/0204301.

[23] J. Alvarez-Muniz and F. Halzen, Phys.Rev. D 63 (2001) 037302.

[24] G. C. Hill and K. Rawlins, Astropart. Phys. 19 (2003) 393.

[25] A. Bouchta et al., http://www.ifh.de/nuastro/software/siegmund/siegmund.html (2000).

[26] S. N. Boziev et al., INR-Preprint 0630 (1989).

[27] D. Heck and J. Knapp, Extensive Air Shower Simulation with CORSIKA: A User's Guide, Forschungszentrum Karlsruhe, Institut für Kernphysik (2000).

[28] C. G. S. Costa, Astropart. Phys. 16 (2001) 193.

[29] T. Gaisser and M. Honda, Ann. Rev. of Nuclear and Particle Science 52 (2002) 153.

[30] G. C. Hill, PhD thesis, University of Adelaide (1996).

[31] A. D. Martin, W. J. Stirling and R. G. Roberts, Phys. Lett. B 354 (1995) 155.

[32] A. M. Dziewonski and D. L. Anderson, Physics of the Earth and Planetary Interiors 25 (1981) 297.

[33] P. Lipari and T. Stanev, Phys. Rev D 44 (1991) 3543.

[34] P. Lipari, Astropart. Phys. 1 (1993) 195.

[35] E. V. Bugaev et al., Phys. Rev. D 58 (1998) 054001.

[36] M. Thunman, G. Ingelman and P. Gondolo, Astropart.Phys. 5 (1996) 309.

[37] J. Ahrens et al., Phys. Rev. D 67 (2003) 012003. 
[38] W. Lohmann, R. Kopp and R. Voss, CERN Yellow Report 85-03 (1985).

[39] A. Karle, Proc. Workshop on Simulation and Analysis Methods for Large Neutrino Telescopes, DESY-PROC-1999-01 (1999) 174.

[40] S. Hundermark, Proc. Workshop on Simulation and Analysis Methods for Large Neutrino Telescopes, DESY-PROC-1999-01 (1999) 276.

[41] S. Hundertmark, PhD thesis, Humboldt-Universität zu Berlin (1999).

[42] V. J. Stenger, DUMAND internal Report HDC-1-90 (1990).

[43] J. Ahrens et al., to be submitted to NIM.

[44] C. Wiebusch, Proc. Workshop on Simulation and Analysis Methods for Large Neutrino Telescopes, DESY-PROC-1999-01 (1999) 302.

[45] G. J. Feldman and R. D. Cousins, Phys. Rev. D 57 (1998) 3873.

[46] Y. Fukuda et al., Phys. Rev. Lett. 81 (1998) 1581.

[47] Q. R. Ahmad et al., Phys. Rev. Lett. 87 (2001) 071301.

[48] E. Waxman and J. Bahcall, Phys. Rev. D 59 (1999) 023002.

[49] K. Mannheim, R. J. Protheroe and J. P. Rachen, Phys. Rev. D 63 (2001) 023003.

[50] F. W. Stecker and M. H. Salamon, Space Sci. Rev. 75 (1996) 341.

[51] E. Waxman, to appear in Nuc. Phys. B, astro-ph/0211358

[52] J. Ahrens et al. , Astrophys. J 583 (2003) 1040. 